\title{
ORIGINAL ARTICLE \\ Fisetin inhibits inflammation and induces autophagy by mediating PI3K/AKT/mTOR signaling in LPS-induced RAW264.7 cells
}

\author{
Yue Sun ',2†, Hong Qin'†, Huihui Zhang', Xiangling Feng', Lina Yang', De-Xing Hou' and \\ jihua Chen ${ }^{\text {** }}$ \\ 'Xiangya School of Public Health, Central South University, Changsha, China; ${ }^{2}$ Inspecting Agency, Shanghai Municipal \\ Health Commission, Shanghai, China; ${ }^{3}$ Course of Biological Science and Technology, The United Graduate School of \\ Agricultural Sciences, Department of Food Science and Biotechnology, Faculty of Agriculture, Kagoshima University, \\ Kagoshima, Japan
}

\section{Popular scientific summary}

- LPS induced inflammatory response and blocked autophagosome-lysosome fusion and degradation in mouse macrophages.

- Fisetin inhibited the expression and secretion of inflammatory cytokines and facilitated autophagosome-lysosome fusion and degradation in LPS-treated RAW264.7 cells via inhibition of the $\mathrm{PI} 3 \mathrm{~K} / \mathrm{AKT} / \mathrm{mTOR}$ signaling pathway.

\section{Abstract}

Background: Fisetin, a natural potent flavonoid, has various beneficial, pharmacological activities. In this study, we investigated expression changes of the fisetin regulating genes in lipopolysaccharide (LPS)-treated RAW264.7 cells and explored the role of fisetin in inflammation and autophagy.

Methods and results: Microarray analysis identified 1,071 genes that were regulated by fisetin in LPS-treated RAW264.7 cells, and these genes were mainly related to the process of immune system response. Quantitative real-time polymerase chain reaction and Bio-Plex analysis indicated that fisetin decreased the expression and secretion of several inflammatory cytokines in cells administered with LPS. Western blot analysis and immunofluorescence assay showed that fisetin decreased microtubule-associated protein 1 light-chain 3B (LC3B) and lysosome-associated membrane protein 1 (LAMP1) expression in LPS-treated cells, while the autophagy inhibitor chloroquine (CQ) could partially reverse this effect. In addition, fisetin reduced the elevated expression of p-PI3K, p-AKT and p-mTOR induced by LPS in a concentration-dependent manner.

Conclusions: Fisetin diminished the expression and secretion of inflammatory cytokines and facilitated autophagosome-lysosome fusion and degradation in LPS-treated RAW264.7 cells via inhibition of the PI3K/ AKT/mTOR signaling pathway. Overall, the results of this study provide new clues for the anti-inflammatory mechanism of fisetin and explain the crosstalk between autophagy and inflammation to some extent.

Keywords: fisetin; macrophage; PI3 KIAKT/mTOR pathway; inflammatory response; autophagy

I nflammation plays an important role in host defenses against invading microorganisms and injury, and it is a significant component in the pathophysiology of many chronic diseases, such as atherosclerosis $(1,2)$, inflammatory bowel disease (3), neurodegenerative diseases, and rheumatoid arthritis $(4,5)$. A coordinated series of common effector mechanisms of inflammation lead to pathological changes in various targeted tissues, such as tissue injury, oxidative stress, remodeling of the extracellular matrix, angiogenesis and fibrosis (6).

Macrophages are important mediators in the immune response against bacterial invasion and participated significantly in the regulation of the inflammatory environment (7). These have a dual role in tissue repair, regeneration

†These authors contributed equally to this work. 
and fibrosis. On the one hand, macrophages promote tissue repair and regeneration by phagocytosing cell debris and pathogen, releasing multiple growth factors, soluble mediators, and anti-inflammatory mediators. On the other hand, macrophage dysfunction could lead to uncontrolled release of inflammatory mediators and growth factors, insufficient production of anti-inflammatory mediators, and failed communication between macrophages and other cell lineages, thereby contributing to chronic inflammation, and eventually leading to tissue fibrosis (8). Lipopolysaccharide (LPS) is an endotoxin released by Gram-negative (G-) bacteria. Generally, LPS binds to toll-like receptor expressed in macrophages and induces the secretion of inflammatory cytokines by activating multiple inflammatory signaling pathways $(9,10)$. It has been reported that LPS translocates to the liver, and activated macrophages play a central role in the development of alcoholic hepatitis. Hao et al. have proved that hepatic ATF4 plays a pathological role in alcohol-induced mitochondrial dysfunction and liver injury by disrupting the nuclear respiratory factor 1 (NRF1)mitochondrial transcription factor A (TFAM) pathway (11).

Autophagy is a catabolic process in which cytoplasmic contents are delivered to lysosomes through doublemembrane autophagosomes for bulk degradation to maintain cellular homeostasis. At present, more than 30 autophagy-related genes and gene products have been identified. The microtubule-associated protein 1 light chain $3 \mathrm{~B}(\mathrm{LC} 3 \mathrm{~B})$ is one of the structural proteins of autophagosomal membranes and is analyzed as a key marker for autophagosomes (12).

Lysosome-associated membrane protein 1 (LAMP1) are major protein components of the lysosomal membrane with a large luminal domain, one transmembrane domain, and a C-terminal cytoplasmic tail. The presence of LAMP1 molecules is one of the major definitions of the lysosomal membrane $(13,14)$. The autophagy regulatory network is very complex, which involved multiple signaling pathways, including TORC1, Ras/PKA, Sch9, AMPK, eIF2 $\alpha$ kinase, and so on (15). It has been proved that autophagy plays a critical role in the differentiation and development of both mammals and invertebrates (16), and functions in tumor suppression (17). There is a bidirectional communication between autophagy and inflammation. Multiple immune mediators induce or suppress autophagy, while a return to homeostasis following a robust immune response is critically dependent on autophagy (18). In fact, autophagy importantly participated in macrophage function (inflammatory response and phagocytosis) (19).

Fisetin, a natural potent flavonoid with a polyphenolic structure, belongs to a class of plant secondary metabolites and is widely found in a variety of fruits and vegetables. It has various pharmacological activities, including anti-inflammatory, anti-apoptotic, anti-mutagenic, antioxidative, anti-carcinogenic and so on (20). Several epidemiological studies have shown that high consumption of dietary flavonoids could reduce the risk of coronary heart disease $(21,22)$, cancer $(23,24)$, stroke $(25)$, and other chronic diseases. The mechanisms of fisetin preventing and inhibiting chronic inflammation-related conditions have also been proved in cell culture and animal models (26).

Although fisetin has been shown to exert antiinflammatory activity by suppressing JNK phosphorylation and $\mathrm{NF}-\kappa \mathrm{B}$ activation in LPS-treated RAW264.7 cells $(27,28)$, its effects on macroautophagy in macrophages are still unknown. In this study, we examined the genes targeted by fisetin in LPS-treated macrophages using a high-throughput technology. Based on the outcomes of microarray analysis, we attempt to explore the effect of fisetin on autophagy and the intrinsic relationship between inflammation and autophagy. In conclusion, we hope to provide further evidence for the mechanism of anti-inflammatory activity of fisetin from the perspective of autophagy.

\section{Materials and methods}

\section{Reagents and antibodies}

Fisetin was purchased from Selleck (Houston, TX) and dissolved in dimethyl sulfoxide (DMSO). LPS and methyl thiazolyl tetrazolium (MTT) were purchased from Sigma-Aldrich (St. Louis, MO). Dulbecco's modified Eagle's medium (DMEM) and fetal bovine serum (FBS) were purchased from Gibco (Grand Island, NY). Penicillinstreptomycin solution $(100 \times)$ and trypsin were purchased from Gen-View (Calimesa, CA). TRIzol reagent was obtained from Thermo Fisher (St. Louis, MO).

Antibodies against the following proteins were used: PI3 kinase p110 $\alpha(\mathrm{C} 73 \mathrm{~F} 8)$ rabbit mAb (\#4249, CST, MA), phospho-PI3 kinase p85 (Tyr458)/p55 (Tyr199) Antibody (\#4228, CST, MA), phospho-mTOR (Ser2448) (D9C2) XP® Rabbit mAb (\#5536, CST, MA), LAMP1 (C54H11) Rabbit mAb (\#3243, CST, MA), AKT monoclonal antibody (60203-2-Ig, Proteintech, IL), phospho-AKT (Ser473) monoclonal antibody (66444-1-Ig, Proteintech, IL), mTOR polyclonal antibody (20657-1-AP, Proteintech, IL), LC3B rabbit pAb (A7198, ABclonal, MA), $\alpha$-tubulin mouse mAb (AC012, ABclonal, MA), horseradish peroxidase (HRP)-conjugated goat anti-mouse IgG (IH-0032, Dingguo, China), and HRP-conjugated goat anti-rabbit IgG $(\mathrm{H}+\mathrm{L})(\mathrm{IH}-0011$, Dingguo, China).

\section{Cell culture}

RAW264.7 cell line, one mouse macrophage cell line, was obtained from the Advanced Research Center of Central South University (Changsha, China). Cells were cultured in DMEM medium supplemented with $10 \%$ FBS and penicillin-streptomycin solution at $37^{\circ} \mathrm{C}$ in a humidified incubator containing $5 \% \mathrm{CO}_{2}$. 


\section{Cell viability assay}

The MTT assay was used to detect the cell viability. Cells were seeded in 96-well plates at a concentration of $5 \times 10^{3}$ cells/well, cultured for $24 \mathrm{~h}$, and subsequently treated with different concentrations of fisetin $(0,5$, $10,20,40,80$, and $160 \mu \mathrm{M})$ for $24 \mathrm{~h}$ or exposed to LPS $(40 \mathrm{ng} / \mathrm{mL})$ for $6 \mathrm{~h}$ after fisetin treatment $(10,20,30 \mu \mathrm{M})$ for $30 \mathrm{~min}$. Afterwards, $110 \mu \mathrm{L}$ MTT solution (MTT powder was dissolved in DMEM at a concentration of $5 \mathrm{mg} /$ $\mathrm{mL}$ ) was added into each well, and cells were incubated for $4 \mathrm{~h}$ at $37^{\circ} \mathrm{C}$. The supernatant was then discarded, and $150 \mu \mathrm{L}$ DMSO solution was added. After 15 min for lowspeed shaking, the absorbance was detected at $490 \mathrm{~nm}$ using a microplate reader (BioTek, Winooski, VT). Cell viability was expressed as a percentage of MTT reduction. Each experiment was repeated four times.

\section{Microarray and bioinformatic analysis}

RAW264.7 cells were pretreated with or without $10 \mu \mathrm{M}$ fisetin for $30 \mathrm{~min}$, and then exposed to $40 \mathrm{ng} / \mathrm{mL}$ LPS for 6 h. Total RNA was extracted using an RNeasy Mini Kit (Qiagen, Valencia, CA) following the manufacturer's protocol. The quantity of RNA was assessed using an Agilent 2100 bioanalyzer (Agilent, Palo Alto, CA). Total RNA (500 ng) was amplified using a low RNA input fluorescent linear amplification kit (Agilent) according to the manufacturer's protocol. Sample cRNA was labeled with cyanine 5 (Cy5), while the universal mouse reference RNA (Agilent) was labeled with cyanine 3 (Cy3). Mouse microarrays (Agilent) containing 22,050 oligonucleotides were used for hybridization following the Agilent microarray processing protocol. Briefly, Cy3- or Cy5-labeled cRNA was mixed and incubated with a microarray slide using an in-situ hybridization kit. After washing and drying, microarray signals were scanned using a Agilent model G2505A microarray scanner. Initial microarray chip images were analyzed using Agilent Feature Extraction software. The cutoff criteria for differentially expressed genes (DEGs) were fold change $\geq$ 2.0 or $\leq 0.5$ between two groups.

The Gene Ontology (GO) knowledgebase is the world's largest source of information on the functions of genes, which classifies gene function into three categories: biological process, molecular function, and cellular component. The GO analysis of DEGs was performed using the Database for Annotation, Visualization and Integration Discovery (DAVID) software, version 6.8 (http://david. abcC.ncifcrf.gov).

\section{Quantitative real-time PCR}

Total RNA was used to synthesize cDNA using an RNA reverse transcription kit (Thermo Fisher, Waltham, MA) following the manufacturer's protocol. Six inflammatory genes (IL-1 $\alpha$, IL-1 $\beta$, IL-6, IL-10, Cc13, and GM-CSF) and $\beta$-actin were quantified by quantitative real-time polymerase chain reaction (qPCR) using a LightCycler 96 (Roche, Basel, Switzerland). Reaction mixtures (10 $\mu \mathrm{L})$ were amplified with 45 cycles of $95^{\circ} \mathrm{C}$ for $10 \mathrm{~s}, 60^{\circ} \mathrm{C}$ for 10 $\mathrm{s}$, and $72^{\circ} \mathrm{C}$ for $10 \mathrm{~s}$. The following primers were used in this study:IL-1 $\alpha, \mathrm{F}: 5^{\prime}$-CCCATGATCTGGAAGAGACCA-3', R: 5'-CAAACTTCTGCCTGACGAGC-3'; IL-1 $\beta$, F: 5'-G CAGTGGTTCGAGGCCTAAT-3', R: 5'-GCTGCTTCA GACACTTGCAC-3'; IL-6, F: 5'-CTCTCTGCAAGA GACTTCCATCC-3', R: 5'-AAGTCTCCTCTCCGGAC TTGT-3'; IL-10, F: 5'-GGCGCTGTCATCGATTTC TC-3', R: 5'-ATGGCCTTGTAGACACCTTGG-3'; Ccl3, F: 5'-TACAGCCGGAAGATTCCACG-3'， R: 5'-GTC AGGAAAATGACACCTGGC-3'; GM-CSF, F: 5'-CA GGGTCTACGGGGCAATTT-3', R: 5'-ACAGTCCGT TTCCGGAGTTG-3'; $\beta$-actin, F: 5'-GATCAAGATC ATTGCTCCTCCTG-3'，R: 5'-AGGGTGTAAAACGC AGCTCA-3'. The $2^{-\Delta \Delta C q}$ method was used to calculate the relative mRNA levels.

\section{Bio-Plex assay and ELISA}

Cell supernatants were collected, and the concentrations of IL-1 $\alpha$, IL-1 $\beta$, IL-6, IL-10, Ccl3 and granulocyte-macrophage colony-stimulating factor (GM-CSF) were assessed using Bio-Plex Pro Mouse Cytokine Panel (Bio-Rad, Hercules, CA). The assay was performed using a Bio-Plex machine (Bio-Plex 200 System, Bio-Rad) following the manufacturer's protocol, and the data were analyzed with the Bio-Plex manager software. Cell supernatants were collected, and the concentrations of IL- 6 and TNF- $\alpha$ were measured with a mouse enzyme linked immunosorbent assay (ELISA) kit (ABclonal, Boston, MA) according to the manufacturer's instructions. The absorbance was detected at $520 \mathrm{~nm}$ using a microplate reader (BioTek, Winooski, VT).

\section{Western blot}

Cells were lysed using radioimmunoprecipitation assay (RIPA) lysis buffer (Beyotime, Shanghai, China) containing $1 \mathrm{mM}$ protease inhibitor cocktail (Bimake, Shanghai, China) and sonicated for $15 \mathrm{~min}$ using an ultrasonic cell disrupter system (SCIENTZ, Ningbo, China). The supernatants were collected by centrifugation at $10,000 \times \mathrm{g}$ for $15 \mathrm{~min}$. Proteins were separated by sodium dodecyl sulfate-polyacrylamide gel electrophoresis, and then blotted onto the polyvinylidene difluoride membranes. Subsequently, the membranes were blocked with 5\% non-fat milk for $1 \mathrm{~h}$ at room temperature, and incubated with primary antibodies overnight at $4^{\circ} \mathrm{C}$ and secondary antibodies for $1 \mathrm{~h}$ at room temperature. Finally, target proteins were detected using a chemiluminescence imaging system (Tanon, Shanghai, China) and quantified by ImageJ software.

\section{Immunofluorescence assay}

Cells were cultured on coverslips in 24 well plates and were sequentially treated with $4 \%$ paraformaldehyde solution 
(Solarbio, Beijing, China) for $40 \mathrm{~min}, 0.1 \%$ Triton-X (Solarbio, Beijing, China) for $20 \mathrm{~min}$, and goat serum (Boster, Wuhan, China) for $1 \mathrm{~h}$. Afterwards, cells were incubated with a mixture of LC3B and LAMP1 primary antibodies overnight at $4^{\circ} \mathrm{C}$, incubated with a secondary antibody mixture for $2 \mathrm{~h}$ at room temperature, and stained with DAPI for $5 \mathrm{~min}$. Finally, slides were mounted with antifade mounting medium (Boster, Wuhan, China) and observed under a confocal laser scanning microscope (Leica, German). Cells without primary antibodies incubation were used as a negative control.

\section{Statistical analysis}

Except for the microarray experiment, other experiments were performed at least three times. Data were expressed as mean \pm SD. Statistical analysis was performed with the unpaired Student's $t$-test or one-way analysis of variance (ANOVA) by SPSS 18.0 (Chicago, IL), and $P<0.05$ was considered to be statistically significant.

Results

\section{Effect of fisetin on cell viability}

To evaluate the cytotoxic effect of fisetin on RAW264.7 cells, MTT assay was performed and the cell viability was examined. Figure 1a shows the growth inhibition rates of RAW264.7 cells treated with different concentrations of fisetin $(5-160 \mu \mathrm{M})$ for $24 \mathrm{~h}$. The inhibitory effect of fisetin on cells was enhanced with the increasing concentrations of fisetin, and the IC50 value of fisetin was estimated to be $42.19 \mathrm{mM}$. Furthermore, we assessed the cell viability in the control group, the LPS $(40 \mathrm{ng} / \mathrm{mL})$ group, the fisetin $(10-30 \mu \mathrm{M})$ and LPS $(40 \mathrm{ng} / \mathrm{mL})$ co-treatment group, and the fisetin $(20 \mu \mathrm{M})$ group. The cell viabilities in these groups were similar without statistical significance (Fig. 1b). Hence, $40 \mathrm{ng} / \mathrm{mL}$ LPS and 10-30 $\mu \mathrm{M}$ fisetin were considered non-cytotoxic and were chosen for further study.

Identification of genes targeted by fisetin in LPS-treated RAW264.7 cells using high-throughput techniques

DNA microarrays were used to detect the gene expression profiles of the control group (Con), the LPS group (LPS), and the fisetin and LPS co-treatment group (FIS +LPS). Compared with the control group, 958 genes were up-regulated and 1,801 genes were down-regulated by LPS (LPS vs. Con, fold change $\geq 2.0$ or $\leq 0.5$ ). Among genes regulated by LPS, 1,071 genes were targeted by fisetin (FIS + LPS vs. LPS, fold change $\geq 2.0$ or $\leq 0.5$ ), of which 359 genes were up-regulated in LPS versus Con and down-regulated in FIS+LPS versus LPS, and 712 genes were up-regulated in LPS versus Con and down-regulated in FIS+LPS versus LPS (Fig. 2a, b and Table 1).

We then performed GO enrichment analysis to explore the function of 1,071 genes that were oppositely regulated by LPS and fisetin. For genes down-regulated in LPS versus Con and up-regulated in FIS+LPS versus LPS, terms with the highest $-\log 10 \mathrm{P}$-value in the biological process, molecular function and cellular component included G-protein coupled receptor signaling pathway, G-protein coupled receptor activity, and plasma membrane, respectively (Fig. 2c). For DEGs

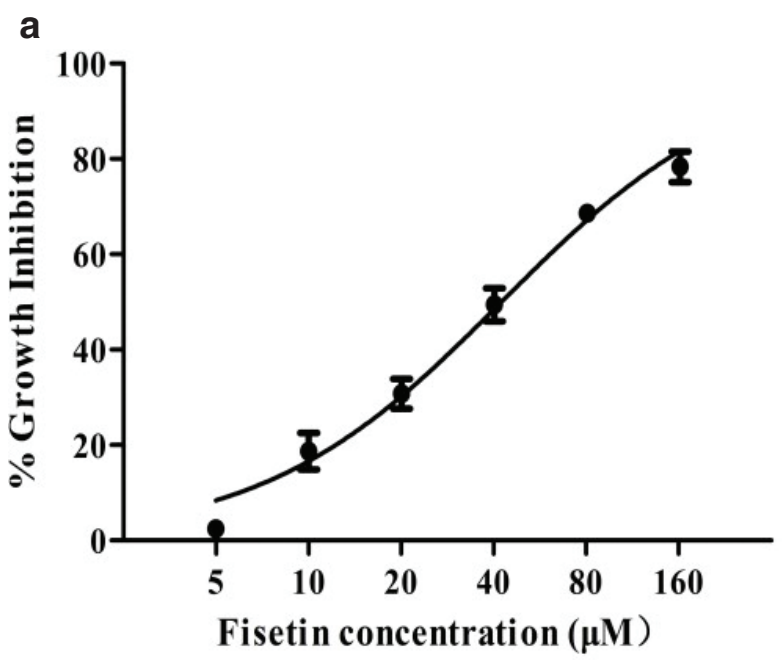

b

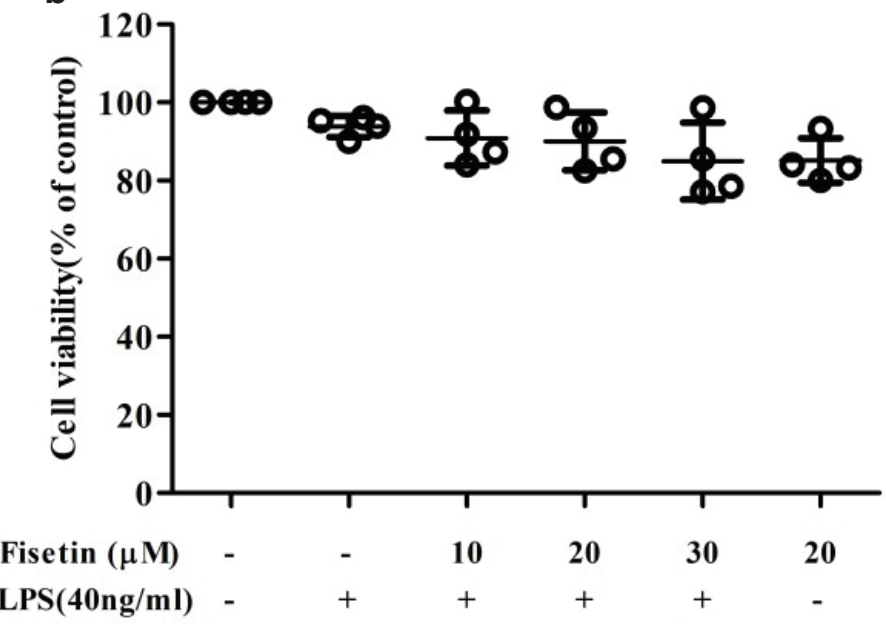

Fig. 1. Effect of fisetin on RAW264.7 cell viability. (a) The growth inhibition rates of RAW264.7 cells treated with different concentrations of fisetin $(5,10,20,40,80$, and $160 \mu \mathrm{M})$ for $24 \mathrm{~h}$; (b) The cell viabilities in the control group, the LPS (40 ng/mL) group, the fisetin $(10-30 \mu \mathrm{M})$ and LPS $(40 \mathrm{ng} / \mathrm{mL})$ co-treatment group, and the fisetin $(20 \mu \mathrm{M})$ group. Cells were treated with fisetin for $24 \mathrm{~h}$, and then exposed to LPS for $6 \mathrm{~h}$. Cell viability was detected by MTT assay, and DMSO treatment was used as a control. Data were presented as mean $\pm \operatorname{SD}(n=4)$. 
a

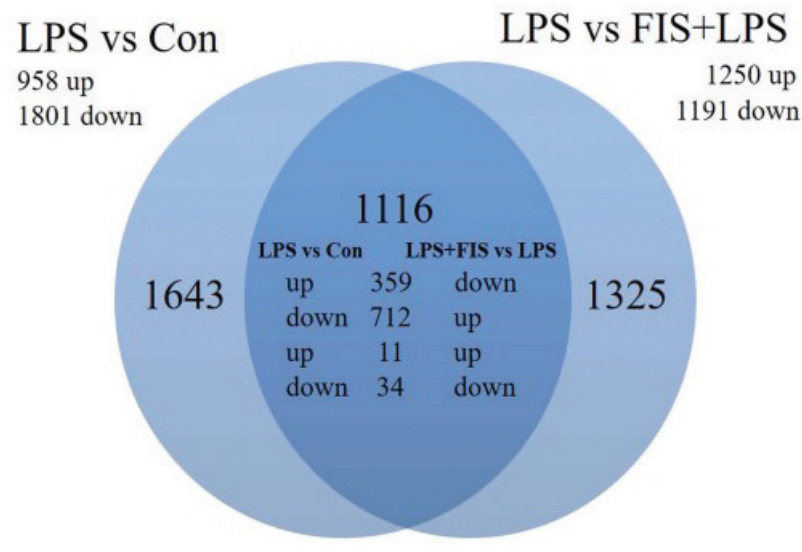

b

Con

LPS FIS+LPS

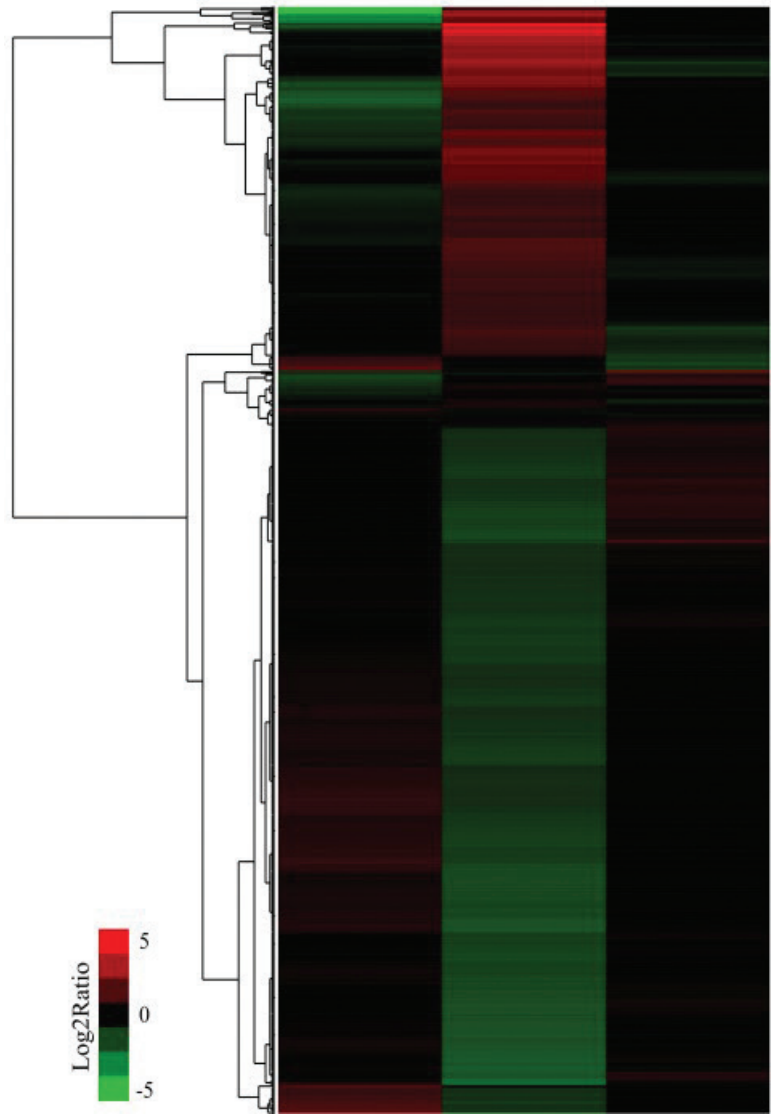

C

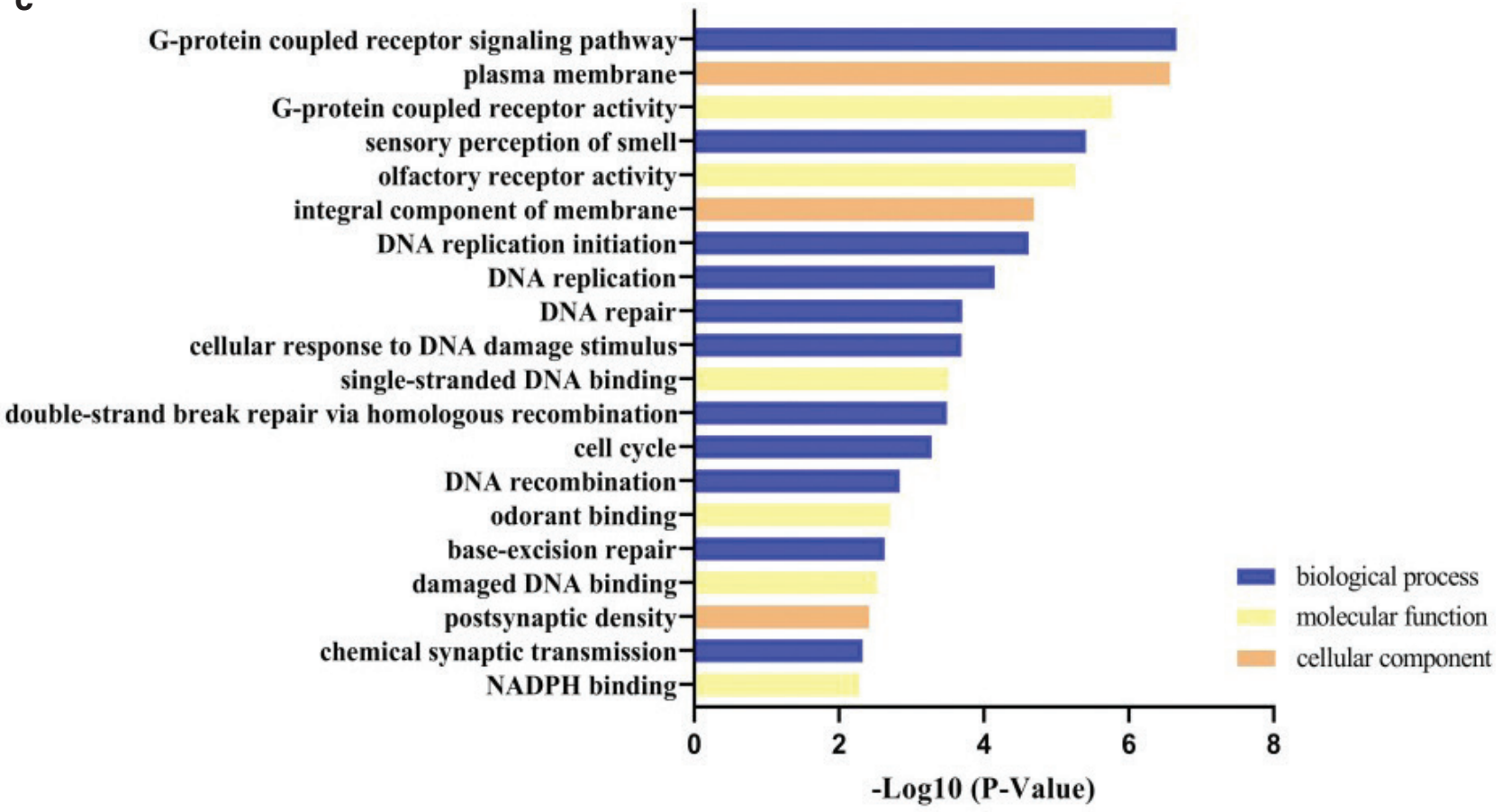

Fig. 2. Microarray analysis of genes targeted by fisetin in LPS-treated RAW264.7 cells. (a) Venn diagram of the number of DEGs in LPS versus Con and LPS versus FIS+LPS; (b) hierarchical clustering of genes targeted by fisetin in LPS-treated RAW264.7 cells; (c) GO enrichment analysis of genes down-regulated in LPS versus Con and up-regulated in FIS+LPS versus LPS, and (d) genes up-regulated in LPS versus Con and down-regulated in FIS+LPS versus LPS. The GO terms were sorted by $-\log 10$ of the enrichment $P$-value. 


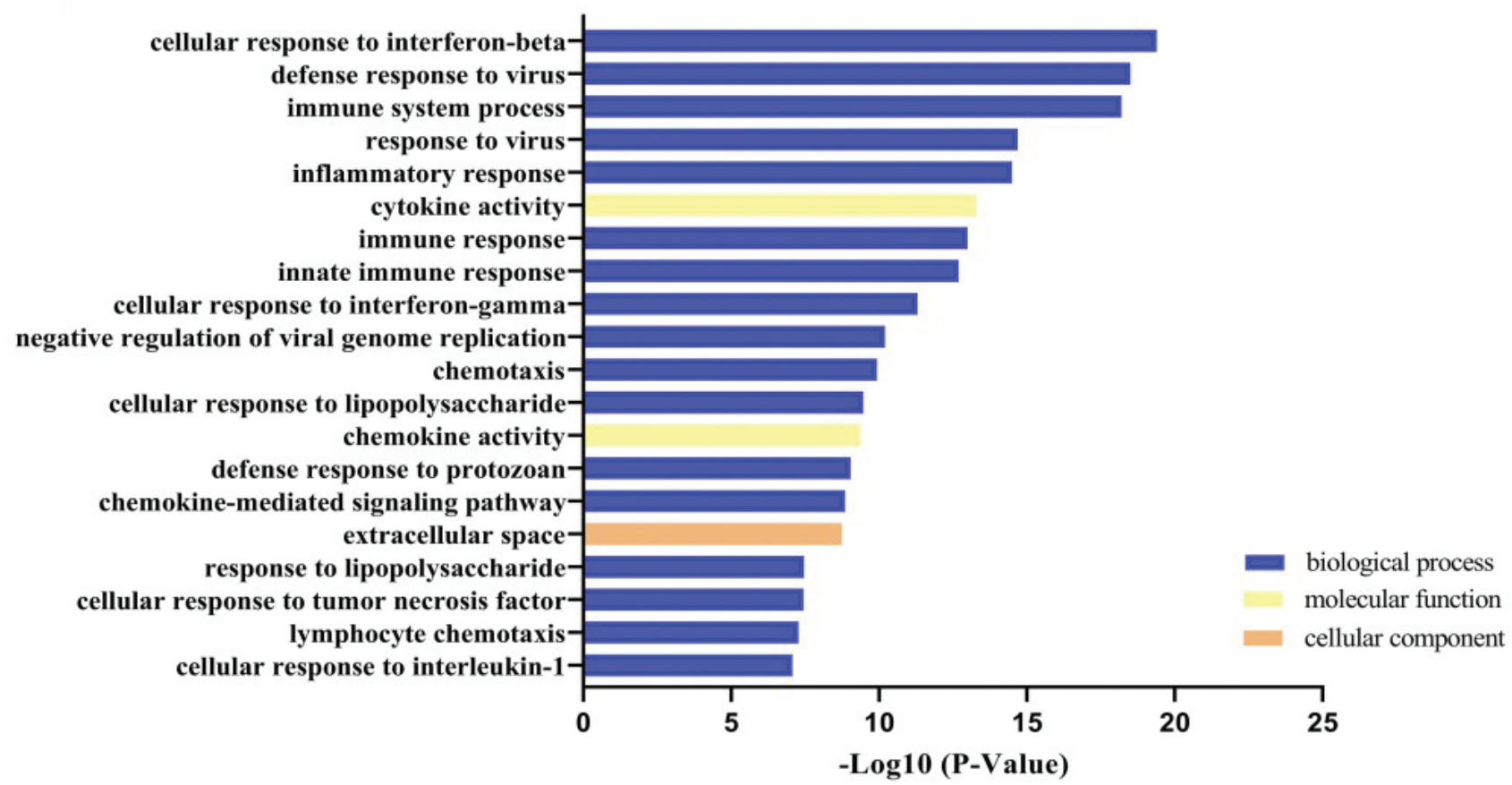

Fig. 2. (Continued) Microarray analysis of genes targeted by fisetin in LPS-treated RAW264.7 cells. (a) Venn diagram of the number of DEGs in LPS versus Con and LPS versus FIS+LPS; (b) hierarchical clustering of genes targeted by fisetin in LPStreated RAW264.7 cells; (c) GO enrichment analysis of genes down-regulated in LPS versus Con and up-regulated in FIS+LPS versus LPS, and (d) genes up-regulated in LPS versus Con and down-regulated in FIS+LPS versus LPS. The GO terms were sorted by $-\log 10$ of the enrichment $P$-value.

Table 1. Number of genes targeted by fisetin (FIS) in lipopolysaccharide (LPS)-treated RAW264.7 cells

\begin{tabular}{|c|c|c|c|c|c|}
\hline \multicolumn{3}{|c|}{ Genes up-regulated by LPS and down-regulated by fisetin } & \multicolumn{3}{|c|}{ Genes down-regulated by LPS and up-regulated by fisetin } \\
\hline $\begin{array}{l}\text { Fold change in LPS } \\
\text { versus Con }\end{array}$ & $\begin{array}{l}\text { Fold change in } \\
\text { FIS+ LPS versus LPS }\end{array}$ & Number of genes & $\begin{array}{l}\text { Fold change in } \\
\text { LPS versus Con }\end{array}$ & $\begin{array}{l}\text { Fold change in } \\
\text { FIS+ LPS versus LPS }\end{array}$ & Number of genes \\
\hline \multirow[t]{3}{*}{$\geq 8$} & $0.25<\sim \leq 0.5$ & 27 & $0.25<\sim \leq 0.5$ & $\geq 8$ & I \\
\hline & $0.125<\sim \leq 0.25$ & 21 & & $4 \leq \sim<8$ & 55 \\
\hline & $\leq 0.125$ & 30 & & $2 \leq \sim<4$ & 460 \\
\hline \multirow[t]{3}{*}{$4 \leq \sim<8$} & $0.25 \sim \leq 0.5$ & 46 & $0.125<\sim \leq 0.25$ & $\geq 8$ & 2 \\
\hline & $0.125<\sim \leq 0.25$ & 32 & & $4 \leq \sim<8$ & 42 \\
\hline & $\leq 0.125$ & $\mathrm{II}$ & & $2 \leq \sim<4$ & 147 \\
\hline \multirow[t]{3}{*}{$2 \leq \sim<4$} & $0.25<\sim \leq 0.5$ & 164 & $\leq 0.125$ & $\geq 8$ & 0 \\
\hline & $0.125<\sim \leq 0.25$ & 27 & & $4 \leq \sim<8$ & 2 \\
\hline & $\leq 0.125$ & I & & $2 \leq \sim<4$ & 3 \\
\hline
\end{tabular}

up-regulated in LPS versus Con and down-regulated in FIS+LPS versus LPS, most terms were significantly enriched in biological processes closely related to immune responses, and terms with the highest $-\log 10 \mathrm{P}$-value in biological process, molecular function and cellular component were cellular response to interferon- $\beta$, cytokine activity, and extracellular region, respectively (Fig. 2d).
Fisetin inhibits gene expression and protein secretion of inflammatory cytokines in LPS-treated RAW264.7 cells

To explore the anti-inflammatory mechanisms of fisetin, we screened the genes targeted by fisetin, which were related to inflammatory response or immune response. Fisetin decreased the expression of multiple inflammatory cytokines, including interleukin family members and chemokines (Fig. 3a). 
To validate the above results, we tested several inflammatory cytokines by q-PCR and Bio-plex. The mRNA levels of IL-1a, IL-1 $\beta$, IL-6, IL-10, Ccl3, and GM-CSF increased after LPS exposure, and fisetin could effectively decrease the expression of these genes (Fig. 3b). Generally, cytokines are secreted into the extracellular region to exert the biological effects. Thus, we measured the protein levels of these inflammatory factors from the supernatants, and results were consistent with the microarray analysis and q-PCR (Fig. 3c). These results suggested that fisetin could inhibit the gene expression and protein secretion of multiple inflammatory cytokines, thereby exerting anti-inflammatory effects.

\section{Fisetin facilitates autophagosome-lysosome fusion and} degradation in LPS-treated RAW264.7 cells

As part of the cellular defense system, autophagy plays an important role in the immune response. We screened the genes targeted by fisetin that were associated with autophagy (Fig. 4a). A total of 36 genes were oppositely

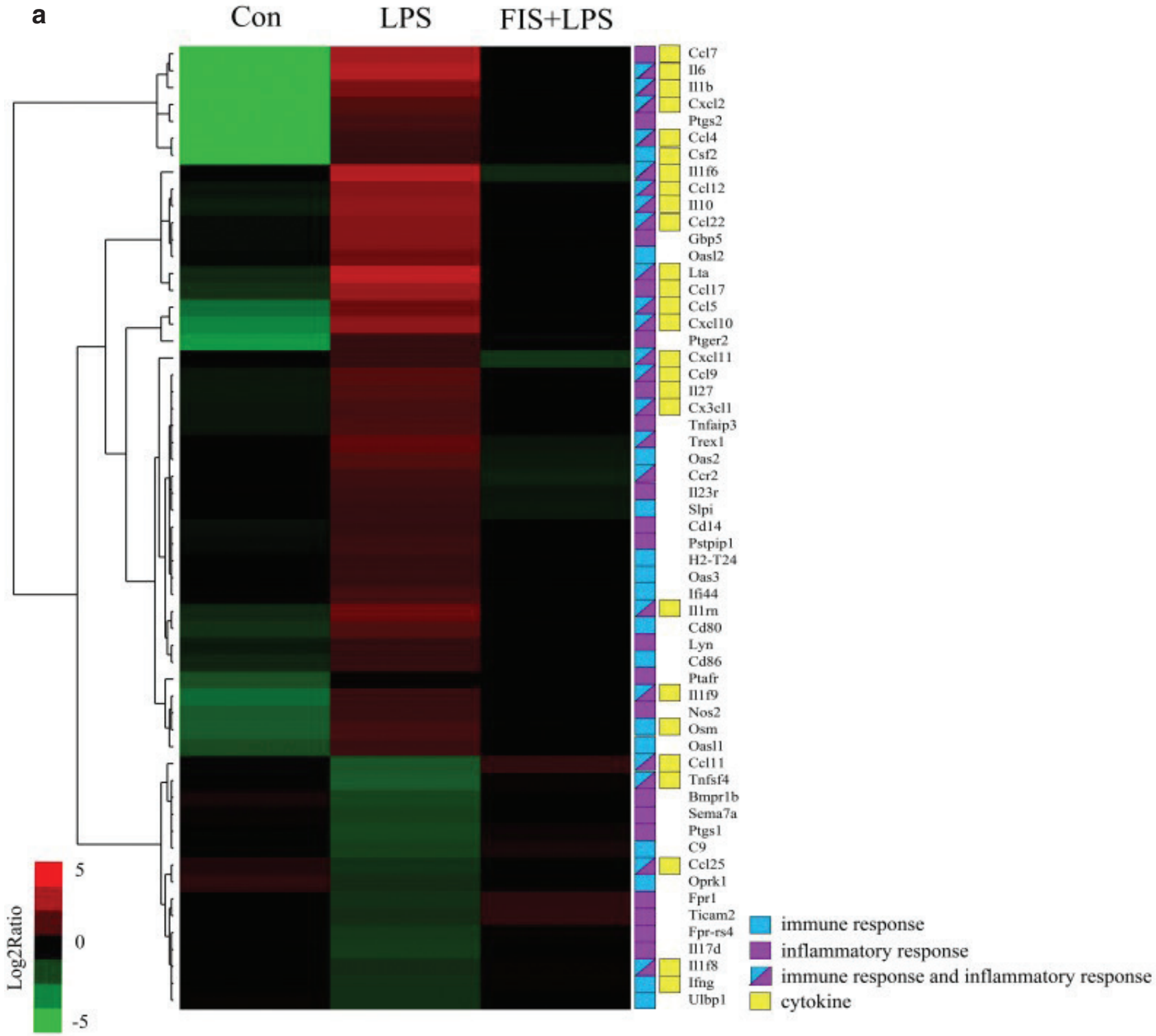

Fig. 3. Effect of fisetin on inflammation in LPS-treated RAW264.7 cells. (a) Hierarchical clustering of DEGs related to inflammatory response or immune response. Log2 ratios were calculated based on $\log 2$ transformed output and input signals. Data were median center across gene, and Euclidean distance and the complete linkage method were used for clustering; (b) the mRNA levels of IL-1a, IL-1 $\beta$, IL-6, IL-10, Cc13 and GM-CSF were detected by qPCR; and (c) secreted proteins of IL-1a, IL-1 $\beta$, IL-6, IL-10, Cc13 and GM-CSF were detected by Bio-plex. Data are represented as mean \pm SD $(n=3)$. $a<0.05$ versus control group; $b<0.05$ versus LPS group. 
b
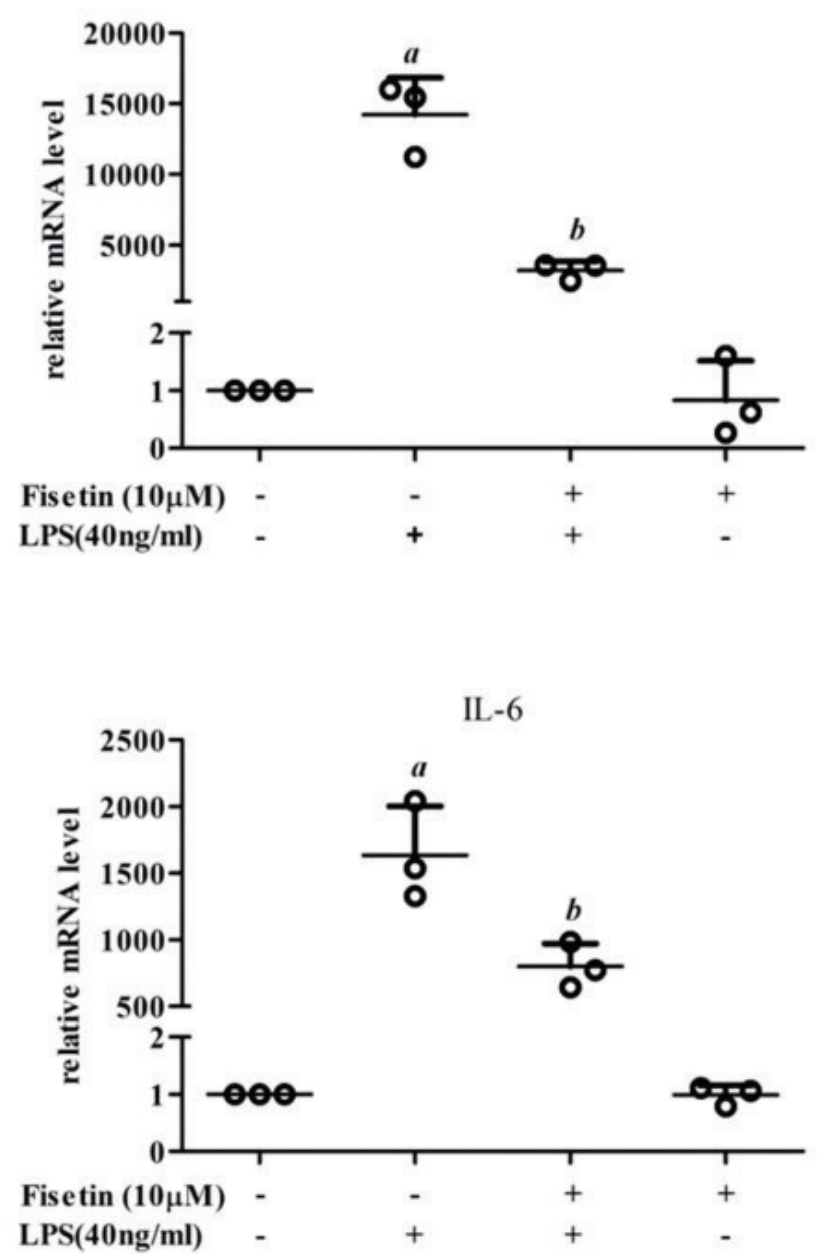

$\mathrm{Ccl} 3$

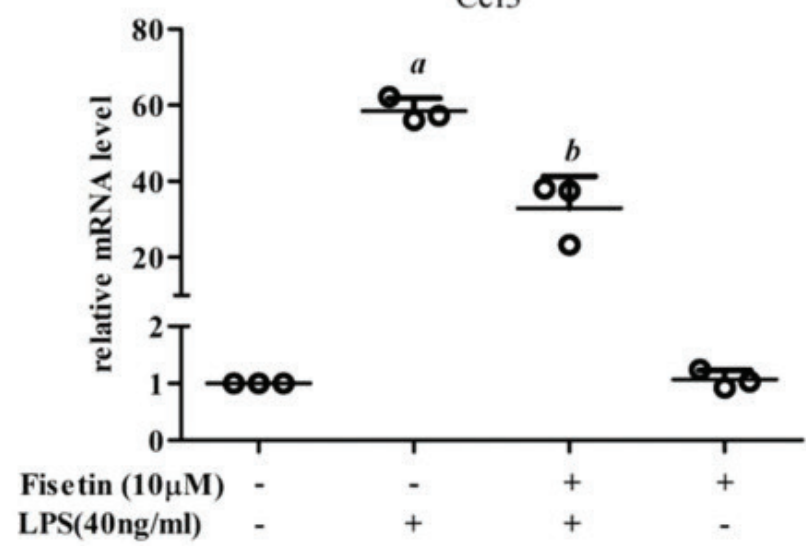

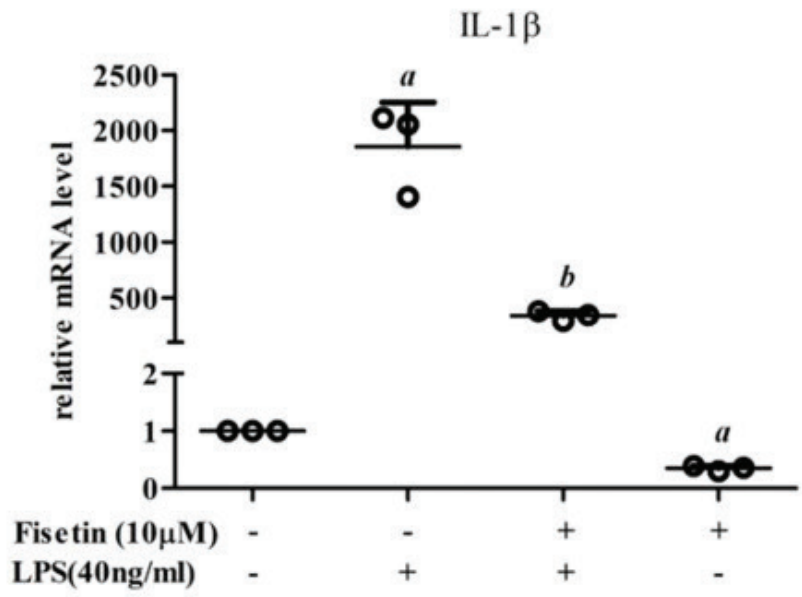
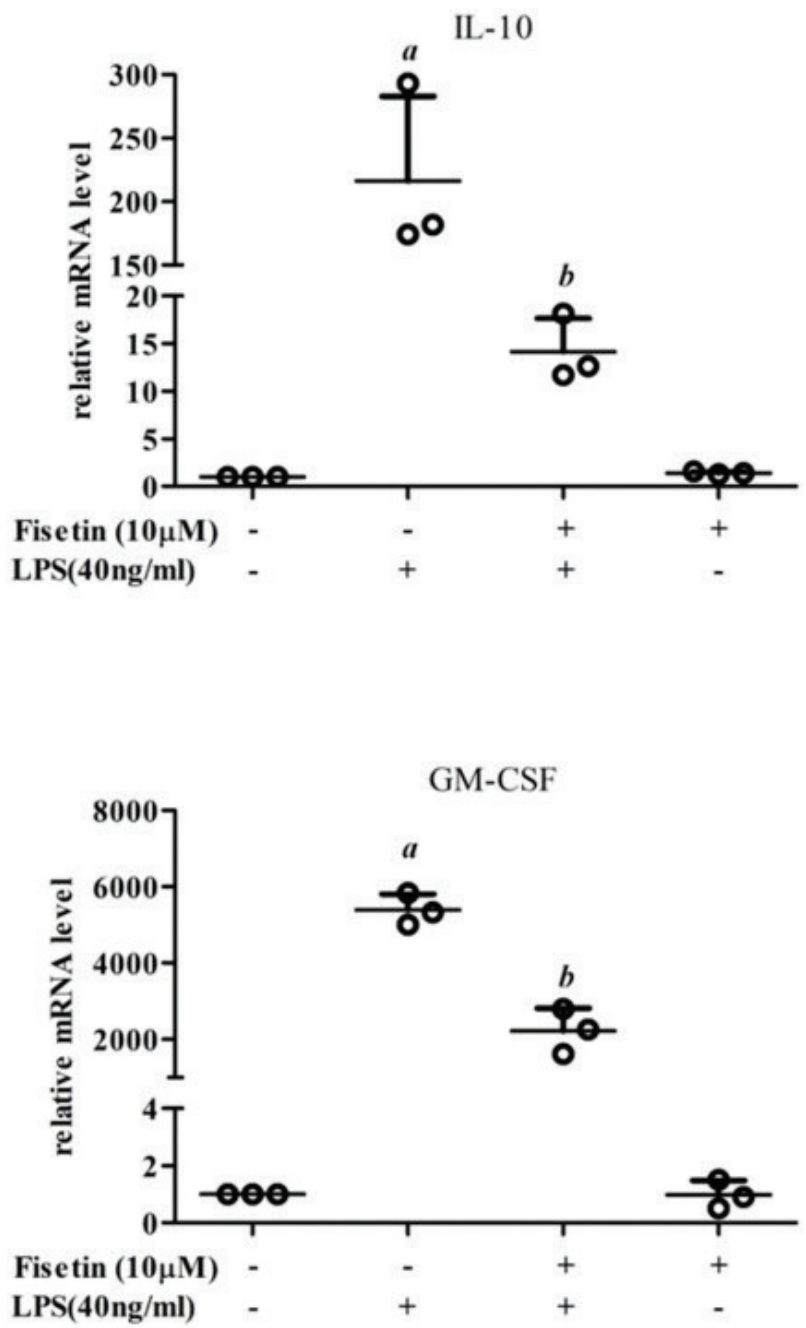

Fig. 3. (Continued) Effect of fisetin on inflammation in LPS-treated RAW264.7 cells. (a) Hierarchical clustering of DEGs related to inflammatory response or immune response. Log2 ratios were calculated based on $\log 2$ transformed output and input signals. Data were median center across gene, and Euclidean distance and the complete linkage method were used for clustering; (b) the mRNA levels of IL-1a, IL-1 $\beta$, IL-6, IL-10, Ccl3 and GM-CSF were detected by qPCR; and (c) secreted proteins of IL-1a, IL-1 $\beta$, IL-6, IL-10, Cc13 and GM-CSF were detected by Bio-plex. Data are represented as mean \pm SD $(n=3)$. $a<0.05$ versus control group; $b<0.05$ versus LPS group. 
c

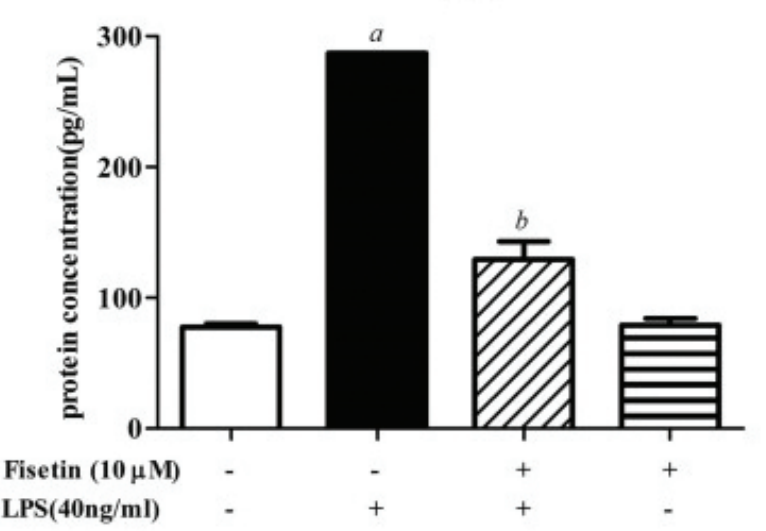

LPS(40ng/ml)
IL-1 $\alpha$

IL-6

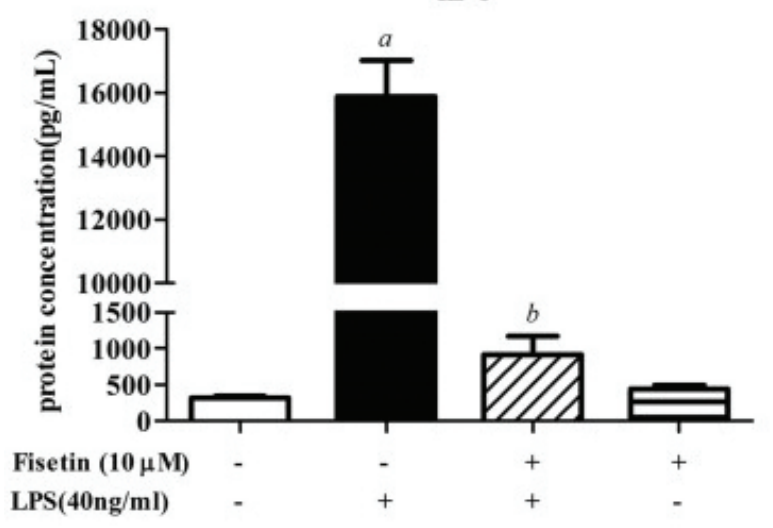

$\mathrm{Ccl} 3$

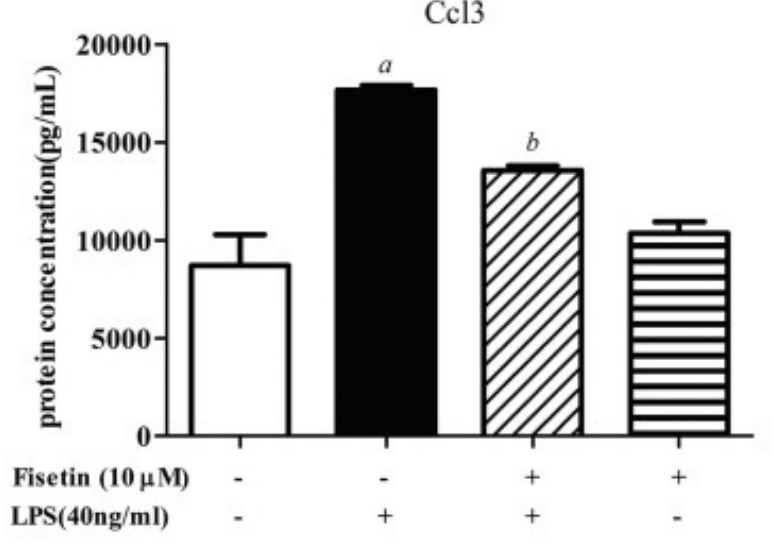

IL-1 $\beta$

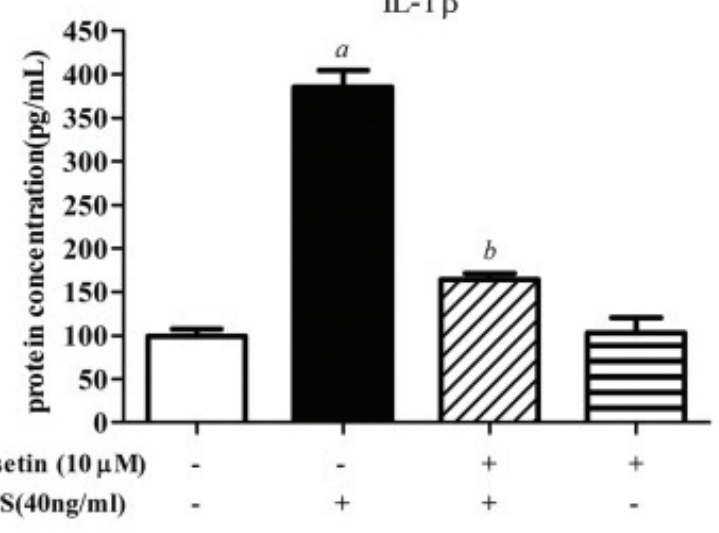

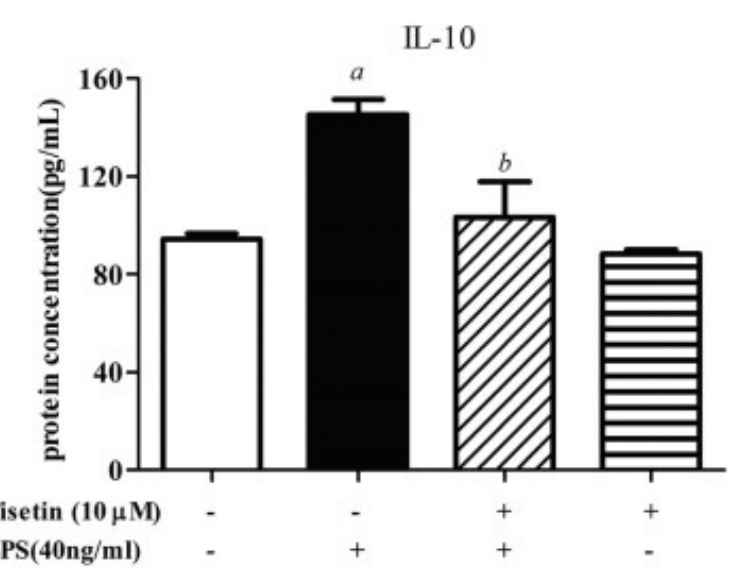

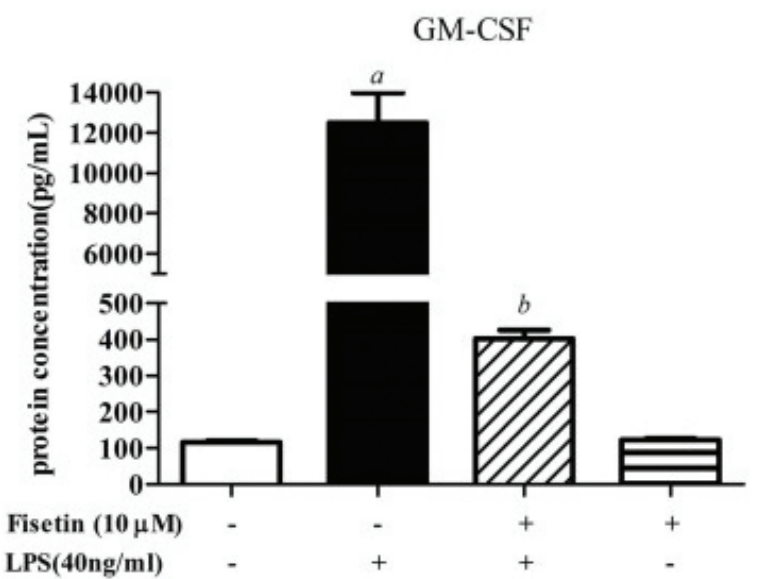

Fig. 3. (Continued) Effect of fisetin on inflammation in LPS-treated RAW264.7 cells. (a) Hierarchical clustering of DEGs related to inflammatory response or immune response. Log2 ratios were calculated based on $\log 2$ transformed output and input signals. Data were median center across gene, and Euclidean distance and the complete linkage method were used for clustering; (b) the mRNA levels of IL-1a, IL-1 $\beta$, IL-6, IL-10, Ccl3 and GM-CSF were detected by qPCR; and (c) secreted proteins of IL-1a, IL-1 $\beta$, IL-6, IL-10, Cc13 and GM-CSF were detected by Bio-plex. Data are represented as mean \pm SD $(n=3)$. $a<0.05$ versus control group; $b<0.05$ versus LPS group. 
a

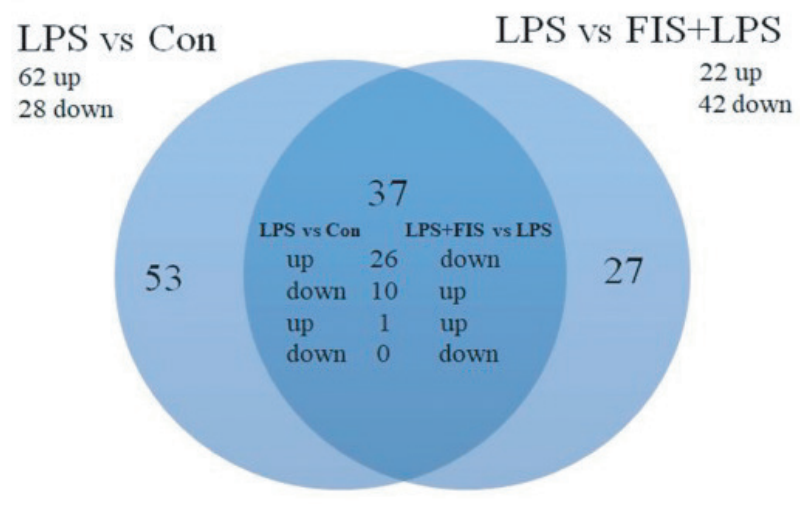

C
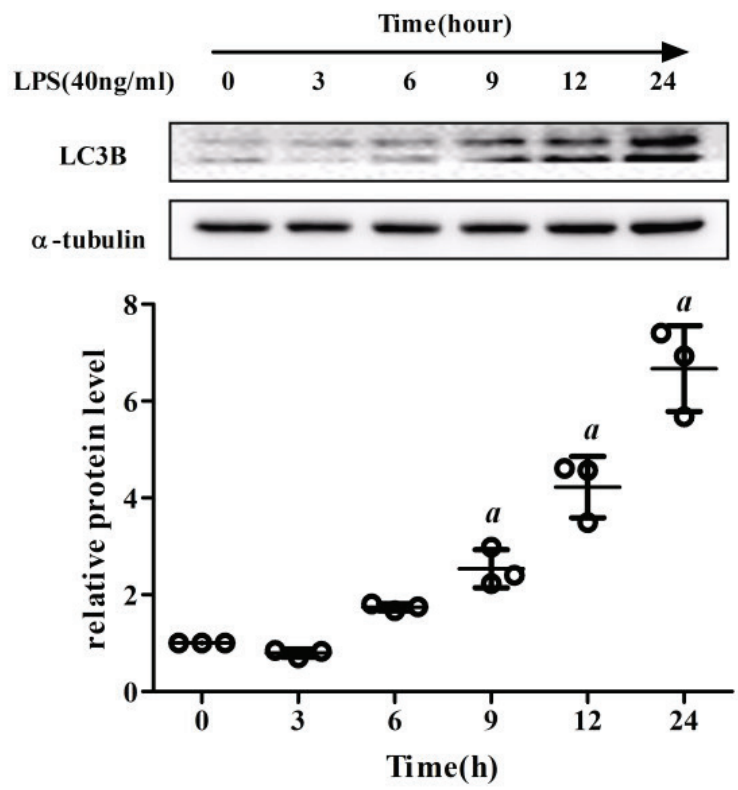

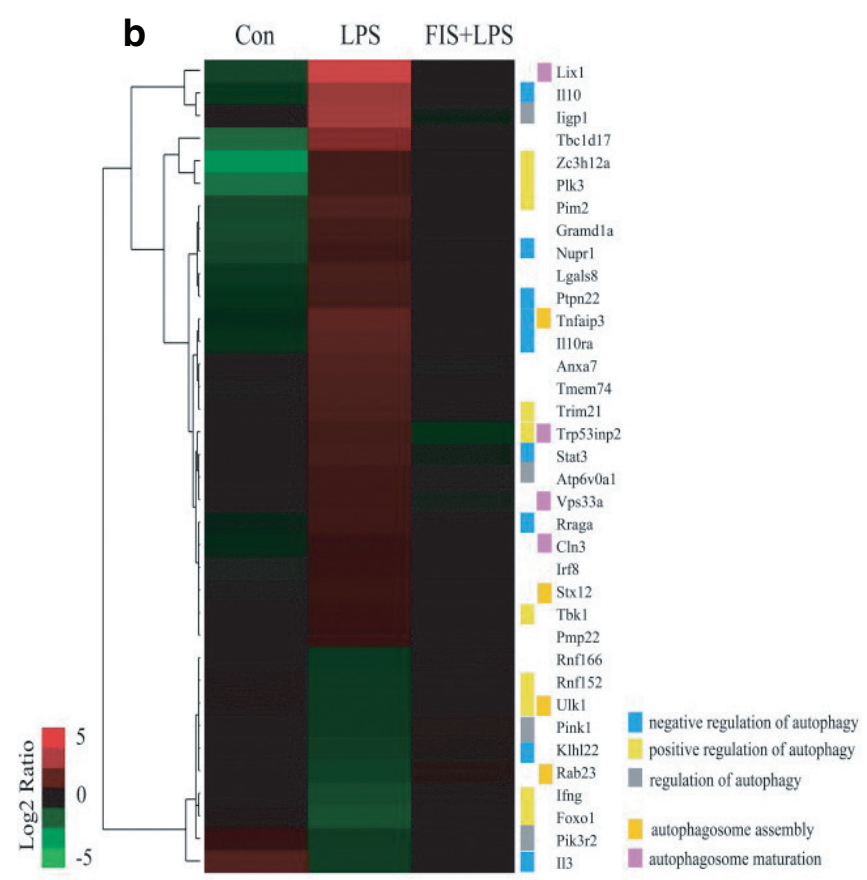

d
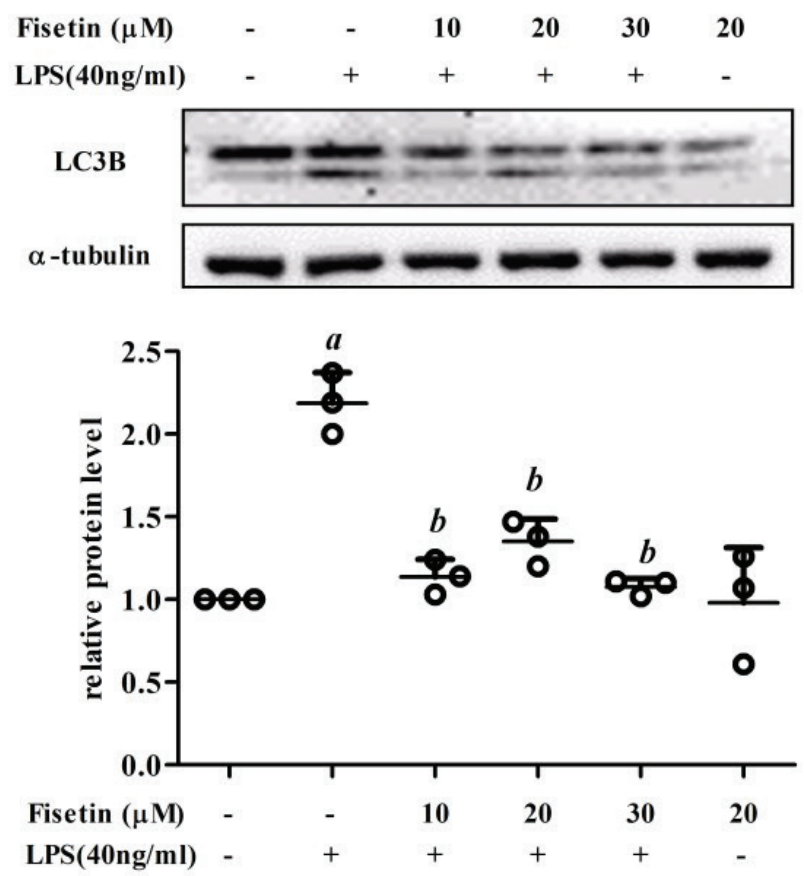

Fig. 4. Effect of fisetin on autophagy in LPS-treated RAW264.7 cells. (a) Venn diagram of the DEGs annotated as the terms associated with autophagy. (b) Hierarchical clustering of the DEGs associated with autophagy. Log2 ratio was calculated based on $\log 2$ transformed output and input signals. Data were median center across gene, and Euclidean distance and the complete linkage method were used for clustering. (c) LC3B and (e) LAMP1 protein levels in cells exposed to LPS for different time periods $(0,3,6,9,12$, and $24 \mathrm{~h})$; (d) LC3B and (f) LAMP1 protein levels in the control group, the LPS (40 ng/mL) group, the fisetin (10, 20 and $30 \mu \mathrm{M})$ and LPS $(40 \mathrm{ng} / \mathrm{mL})$ co-treatment group, and the fisetin $(20 \mu \mathrm{M})$ group. $a<0.05$ versus control group; $b<0.05$ versus LPS group; (g) immunofluorescence of LC3 and LAMP1 in the control group (Control), the LPS group (LPS), the fisetin $(20 \mu \mathrm{M})$ and LPS co-treatment group (FIS+LPS), and the negative control group (Blank); (h) LC3B and (i) LAMP1 protein levels in cells pretreated with CQ. $a<0.05$. Data are represented as mean $\pm \operatorname{SD}(n=3)$. 
e
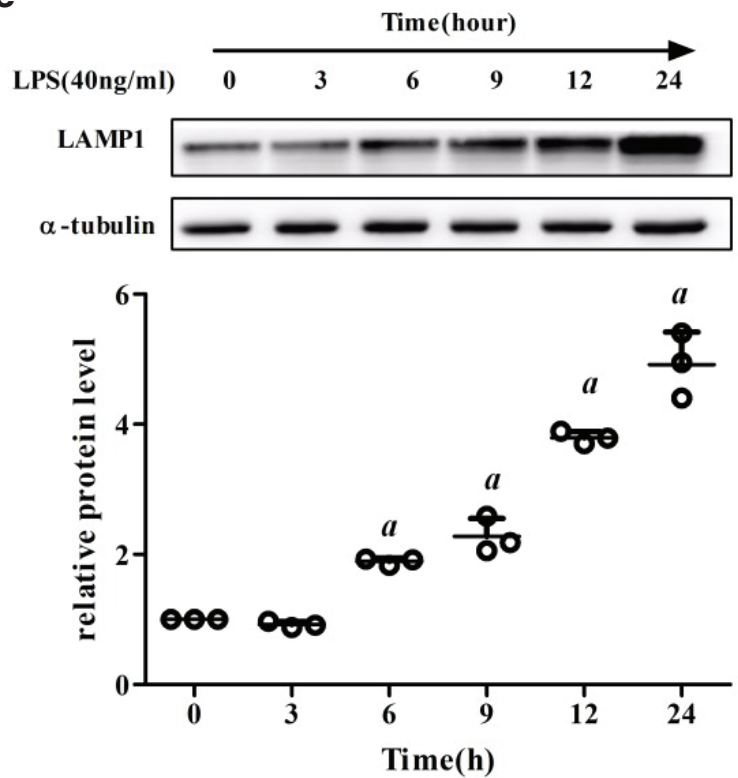

f

$\begin{array}{lllllll}\text { Fisetin }(\mu M) & - & - & 10 & 20 & 30 & 20 \\ \operatorname{LPS}(40 \mathrm{ng} / \mathrm{ml}) & - & + & + & + & + & -\end{array}$

g
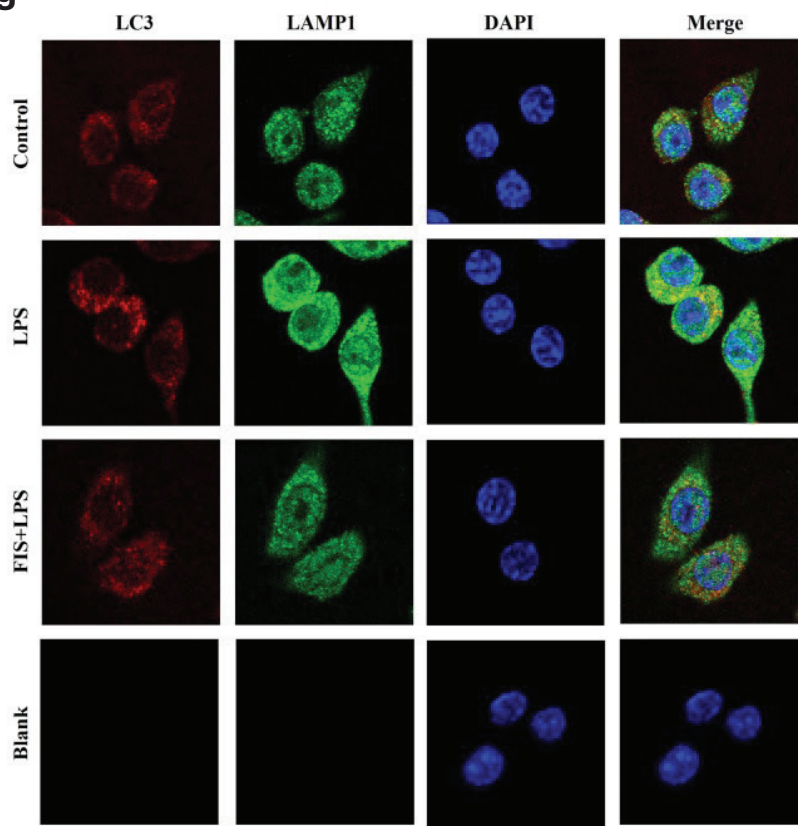

Fig. 4. (Continued) Effect of fisetin on autophagy in LPS-treated RAW264.7 cells. (a) Venn diagram of the DEGs annotated as the terms associated with autophagy. (b) Hierarchical clustering of the DEGs associated with autophagy. Log2 ratio was calculated based on $\log 2$ transformed output and input signals. Data were median center across gene, and Euclidean distance and the complete linkage method were used for clustering. (c) LC3B and (e) LAMP1 protein levels in cells exposed to LPS for different time periods $(0,3,6,9,12$, and $24 \mathrm{~h})$; (d) LC3B and (f) LAMP1 protein levels in the control group, the LPS $(40 \mathrm{ng} / \mathrm{mL})$ group, the fisetin $(10,20$ and $30 \mu \mathrm{M})$ and LPS $(40 \mathrm{ng} / \mathrm{mL})$ co-treatment group, and the fisetin $(20 \mu \mathrm{M})$ group. $a<0.05$ versus control group; $b<0.05$ versus LPS group; (g) immunofluorescence of LC3 and LAMP1 in the control group (Control), the LPS group (LPS), the fisetin $(20 \mu \mathrm{M})$ and LPS co-treatment group (FIS+LPS), and the negative control group (Blank); (h) LC3B and (i) LAMP1 protein levels in cells pretreated with CQ. $a<0.05$. Data are represented as mean \pm SD $(n=3)$. 
h

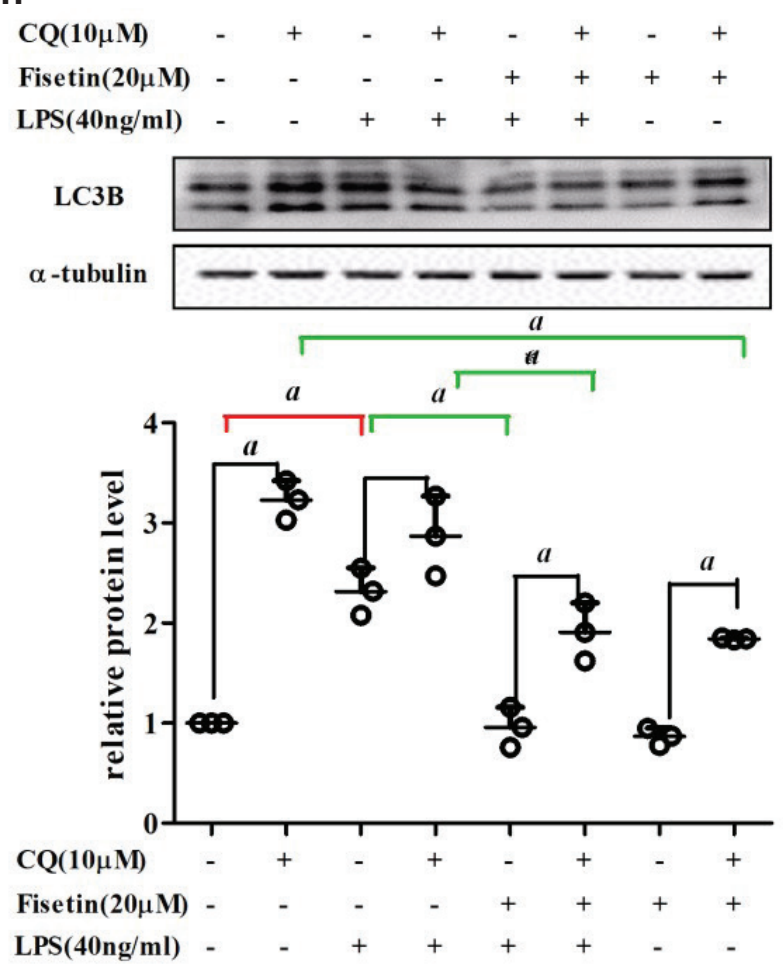

i

$\begin{array}{lllllllll}\operatorname{CQ}(10 \mu \mathrm{M}) & - & + & - & + & - & + & - & + \\ \text { Fisetin }(20 \mu \mathrm{M}) & - & - & - & - & + & + & + & + \\ \operatorname{LPS}(40 \mathrm{ng} / \mathrm{ml}) & - & - & + & + & + & + & - & -\end{array}$

LAMP1

$\alpha-$ tubulin
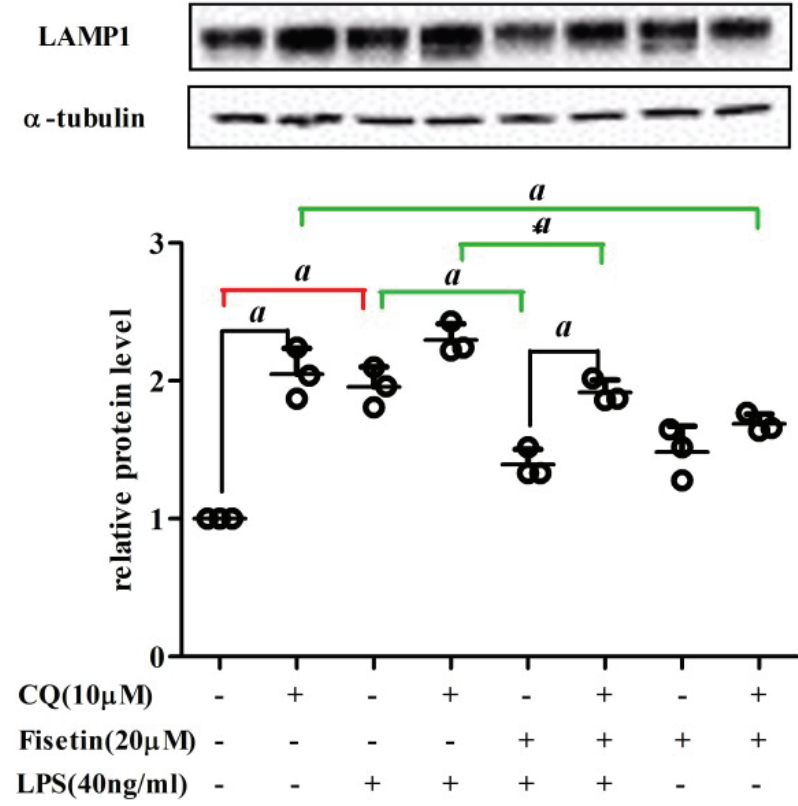

Fig. 4. (Continued) Effect of fisetin on autophagy in LPS-treated RAW264.7 cells. (a) Venn diagram of the DEGs annotated as the terms associated with autophagy. (b) Hierarchical clustering of the DEGs associated with autophagy. Log2 ratio was calculated based on $\log 2$ transformed output and input signals. Data were median center across gene, and Euclidean distance and the complete linkage method were used for clustering. (c) LC3B and (e) LAMP1 protein levels in cells exposed to LPS for different time periods $(0,3,6,9,12$, and $24 \mathrm{~h})$; (d) LC3B and (f) LAMP1 protein levels in the control group, the LPS (40 ng/mL) group, the fisetin $(10,20$ and $30 \mu \mathrm{M})$ and LPS $(40 \mathrm{ng} / \mathrm{mL})$ co-treatment group, and the fisetin $(20 \mu \mathrm{M})$ group. $a<0.05$ versus control group; $b<0.05$ versus LPS group; (g) immunofluorescence of LC3 and LAMP1 in the control group (Control), the LPS group (LPS), the fisetin $(20 \mu \mathrm{M})$ and LPS co-treatment group (FIS+LPS), and the negative control group (Blank); (h) LC3B and (i) LAMP1 protein levels in cells pretreated with CQ. $a<0.05$. Data are represented as mean $\pm \mathrm{SD}(n=3)$.

regulated by LPS and fisetin, and these genes mainly participated in the regulation of autophagy, autophagosome assembly, and maturation (Fig. 4b). Then we investigated the transcriptional changes of macroautophagy molecular markers. Notably, SQSTM1 decreased and Map1lc3b increased in LPS versus Con, which indicated that autophagy flux might be inhibited by LPS (Table 2).

To clarify the effects of fisetin and LPS on autophagy, we detected autophagy marker molecules LC3B and LAMP1 by Western blot analysis. We first examined LC3B protein levels of cells exposed to LPS for different time periods $(0,3,6,9,12$, and $24 \mathrm{~h})$. It increased in a time-dependent manner and showed significant differences after LPS exposure for $9 \mathrm{~h}$ (Fig. 4c). Different concentrations of fisetin could significantly inhibit LC3B expression in
LPS-treated cells (Fig. 4d). LAMP1, an important marker protein of lysosome, also increased in a time-dependent manner and increased significantly after LPS exposure for $6 \mathrm{~h}$ (Fig. 4e). Treatment of different concentrations of fisetin decreased LAMP1 expression in LPS-treated cells (Fig. 4f). Similarly, immunofluorescence assay showed that the expressions of LC3B and LAMP were increased in the LPS group, while fisetin decreased the elevated levels of LC3B and LAMP1 induced by LPS (Fig. 4g).

To verify whether autophagy was inhibited or stimulated, cells were pretreated with chloroquine (CQ), an autophagy inhibitor that could effectively inhibit lysosomal digestion. As shown in Fig. 4h and i, CQ remarkably increased LC3B and LAMP1 accumulation. For cells without CQ pretreatment, LC3B and LAMP1 increased 
Table 2. Fold changes in lipopolysaccharide (LPS) versus control group (Con) and fisetin (FIS)+LPS versus LPS for the macroautophagy molecular markers according to microarray analysis

\begin{tabular}{|c|c|c|c|c|}
\hline \multirow[t]{2}{*}{ Associated process } & \multirow[t]{2}{*}{ Gene name } & \multirow[t]{2}{*}{ Protein name } & \multicolumn{2}{|c|}{ Fold change } \\
\hline & & & LPS versus Con & FIS+LPS versus LPS \\
\hline \multirow{4}{*}{$\begin{array}{l}\text { The Unc-5I-like kinase } \\
\text { (ULK) complex }\end{array}$} & ULKI & Serine/threonine-protein kinase ULKI & 0.57 & 1.64 \\
\hline & ULK2 & Serine/threonine-protein kinase ULK2 & 1.04 & 0.90 \\
\hline & ATGI3 & Autophagy-related protein 13 & 1.15 & 0.76 \\
\hline & Rblccl & RBI-inducible coiled-coil protein I & 1.42 & 0.90 \\
\hline \multirow[t]{2}{*}{ The PI3K complex } & BECNI & Beclin-I & 1.21 & 1.20 \\
\hline & ATGI4 & Beclin I-associated autophagy-related key regulator & 0.67 & 1.75 \\
\hline \multirow{10}{*}{$\begin{array}{l}\text { ATG8/LC3 conjugation } \\
\text { system }\end{array}$} & Mapllc3b & Microtubule-associated proteins IA/IB light chain 3B & 0.69 & 1.45 \\
\hline & Mapllc3a & Microtubule-associated proteins IA/IB light chain $3 \mathrm{~A}$ & 1.28 & 0.95 \\
\hline & Gabarap & Gamma-aminobutyric acid receptor-associated protein & 1.08 & 0.93 \\
\hline & Gabarapll & Gamma-aminobutyric acid receptor-associated protein-like I & 1.43 & 1.23 \\
\hline & Gabarapl2 & Gamma-aminobutyric acid receptor-associated protein-like 2 & 1.15 & 0.98 \\
\hline & Atg4b & Cysteine protease ATG4B & 1.16 & 0.95 \\
\hline & Atg4a & Cysteine protease ATG4A & 1.14 & 0.78 \\
\hline & Atg4d & Cysteine protease ATG4D & 1.01 & 1.10 \\
\hline & Atg7 & Ubiquitin-like modifier-activating enzyme ATG7 & 1.12 & 0.75 \\
\hline & Atg3 & Ubiquitin-like-conjugating enzyme ATG3 & 1.74 & 1.16 \\
\hline \multirow{5}{*}{$\begin{array}{l}\text { ATG I } 2 \text { conjugation } \\
\text { system }\end{array}$} & Atg12 & Ubiquitin-like protein ATG I 2 & 1.16 & 1.64 \\
\hline & Atg7 & Ubiquitin-like modifier-activating enzyme ATG7 & 1.12 & 0.75 \\
\hline & Atg10 & Ubiquitin-like-conjugating enzyme ATG I0 & 0.51 & 1.09 \\
\hline & Atg5 & Autophagy protein 5 & 0.90 & 1.00 \\
\hline & Atg 1611 & Autophagy-related protein 16-I & 1.12 & 1.39 \\
\hline \multirow[t]{2}{*}{ Lysosome } & LAMPI & Lysosome-associated membrane glycoprotein I & 0.95 & I.II \\
\hline & LAMP2 & Lysosome-associated membrane glycoprotein 2 & 1.14 & 1.08 \\
\hline Autophagy substrates & SQSTMI & Ubiquitin-binding protein p62 & 5.06 & 0.85 \\
\hline
\end{tabular}

in the LPS group compared with the control group, and decreased by 0.57 times and 0.29 times after fisetin treatment, respectively. For cells with CQ pretreatment, LC3B and LAMP1 in the LPS group had no significant change, and decreased by about 0.33 times and 0.17 times after fisetin treatment, respectively. These results suggested that LPS blocked the fusion and degradation of autophagosome-lysosome, and treatment of fisetin could recover this process to some extent.

\section{Fisetin inhibits the activation of PI3K/AKT/mTOR signaling pathway in LPS-treated RAW264.7 cells}

To explore the possible mechanisms that fisetin facilitated autophagy in LPS-treated RAW264.7 cells, we examined the critical proteins of PI3K/AKT/mTOR signaling pathway. The protein levels of p-PI3K, p-AKT, and p-mTOR increased in a time-dependent manner in cells exposed to LPS for different time periods, and the total protein level of PI3K, AKT, and mTOR showed no significant differences (Fig. 5a). Treatment of different concentrations of fisetin decreased the protein levels of p-PI3K, p-AKT, and p-mTOR in LPS-treated cells (Fig. 5b). These results indicated that fisetin promoted autophagy by inhibiting the PI3K/AKT/mTOR signaling pathway.

\section{Effect of CQ on the anti-inflammatory property of fisetin}

To explore the correlation between autophagy activation and anti-inflammatory response induced by fisetin, interleukin-6 (IL-6) and tumor necrosis factor-alpha (TNF$\alpha$ ) were measured in the presence or absence of CQ. As shown in Fig. 6, for cells without CQ pretreatment, secretory IL-6 and TNF- $\alpha$ levels increased in the LPS group, while these decreased by about 0.51 and 0.40 times after fisetin treatment, respectively. For cells with CQ pretreatment, secretory IL- 6 and TNF- $\alpha$ levels increased in the LPS group, while these decreased by 0.75 and 0.48 times after fisetin treatment, respectively. Compared with cells without CQ pretreatment, IL-6 in the control group, the 
a
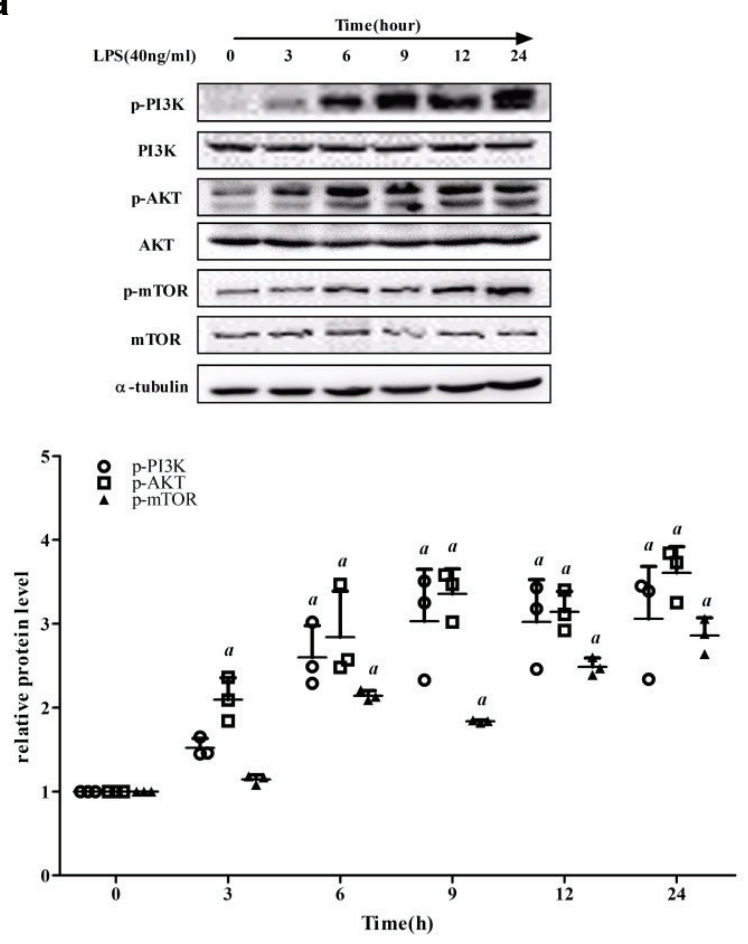

b

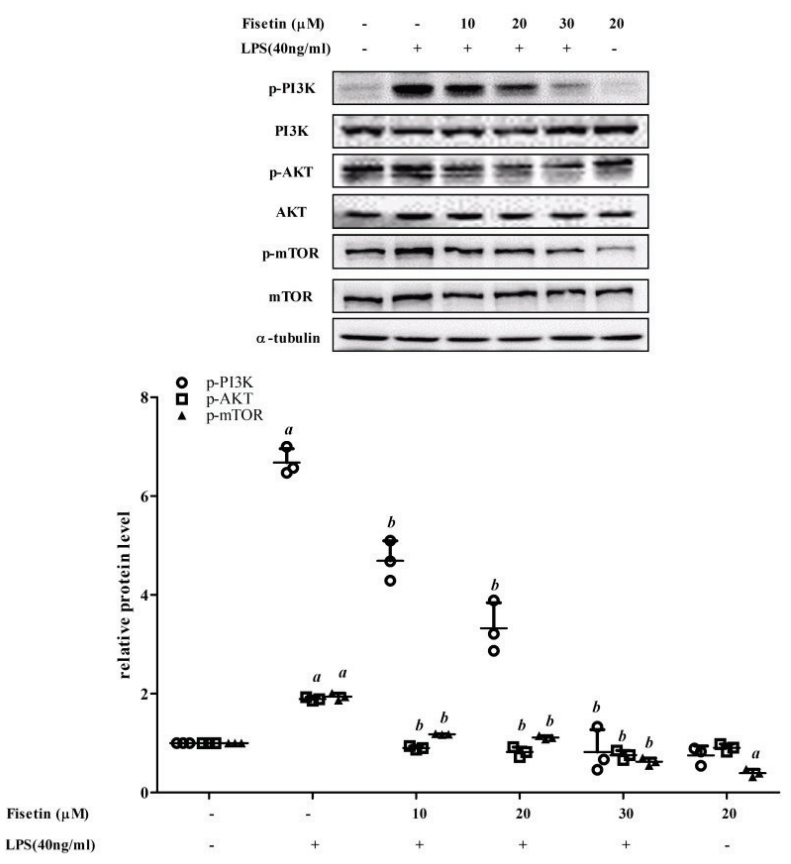

Fig. 5. Effect of fisetin on the PI3K/AKT/mTOR signaling pathway. (a) The protein levels of p-PI3K, p-AKT and p-mTOR in cells exposed to LPS for different time periods $(0,3,6,9,12$, and $24 \mathrm{~h})$. (b) The protein levels of p-PI3K, p-AKT and p-mTOR levels in the control group, the LPS $(40 \mathrm{ng} / \mathrm{mL})$ group, the fisetin $(10,20$ and $30 \mu \mathrm{M})$ and LPS $(40 \mathrm{ng} / \mathrm{mL})$ co-treatment group, and the fisetin $(20 \mu \mathrm{M})$ group. Data are represented as mean $\pm \mathrm{SD}(n=3) . a<0.05$ versus control group; $b<0.05$ versus LPS group.
LPS group, and the fisetin and LPS co-treatment group decreased by $0.55,0.51$, and 0.75 times in cells with CQ pretreatment, respectively. Similarly, levels of TNF- $\alpha$ had decreased by $0.26,0.08$, and 0.15 times in the control group, LPS group, and fisetin and LPS co-treatment group, respectively. For the fisetin group, levels of TNF- $\alpha$ increased in CQ-pretreated cells compared with those without CQ pretreatment, while levels of IL-6 did not change significantly.

\section{Discussion}

Inflammation is a natural process that protects the host from tissue damage and infections, which contributes to the restoration and maintenance of tissue homeostasis. However, persistent inflammation can lead to tissue damage and dysfunction, and underlies the pathogenesis of various chronic diseases. Fisetin could inhibit or reverse the adverse inflammatory response, and is used to prevent or treat several diseases associated with persistent inflammation.

In this study, RAW 264.7 macrophages were exposed to LPS, which is considered as a classic model in inflammation research (29). Microarray analysis was performed to identify the fisetin targeting genes in LPS-treated RAW264.7 cells. We identified the DEGs in LPS versus control and FIS+LPS versus LPS, respectively. The overlapped DEGs with an opposite trend were considered as genes targeted by fisetin in LPS-treated RAW264.7 cells. GO enrichment analysis results indicated the DEGs were significantly involved in the biological process of immune system response. For DEGs that were up-regulated by LPS and down-regulated by fisetin, they were significantly enriched in terms associated with cellular response to cytokine (biological process), cytokine activity (molecular function), and extracellular space (cellular component). The results of q-PCR and bio-plex showed that fisetin decreased mRNA transcription and protein secretion of several inflammatory cytokines (IL-1a, IL-1 1 , IL-6, IL-10, Ccl3, and GM-CSF). These findings were consistent with several previous studies in vitro and in vivo that fisetin inhibited the production of pro-inflammatory mediators through suppression of $\mathrm{NF}-\mathrm{\kappa B}, \mathrm{JNK}$, and other immune-related signaling pathways in LPS-treated macrophages $(28,30,31)$, and exerted anti-inflammatory activity in LPS-induced colitis (32), acute lung injury (33), central nervous system-insult (34), and acute otitis (35). It is worth noting that LPS up-regulated and fisetin down-regulated the mRNA and protein levels of IL-10, a well-known anti-inflammatory cytokine that plays a crucial role in preventing inflammatory and autoimmune pathologies.

However, aberrant expression of IL-10 can not only enhance inflammatory response to microbial challenge but also lead to development of inflammatory bowel disease and a number of autoimmune diseases (36). 
a

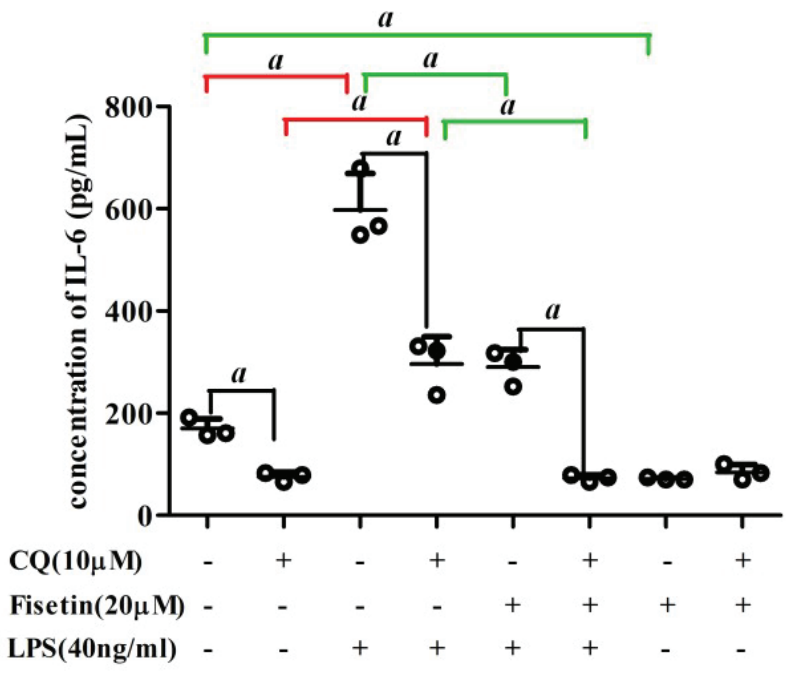

b

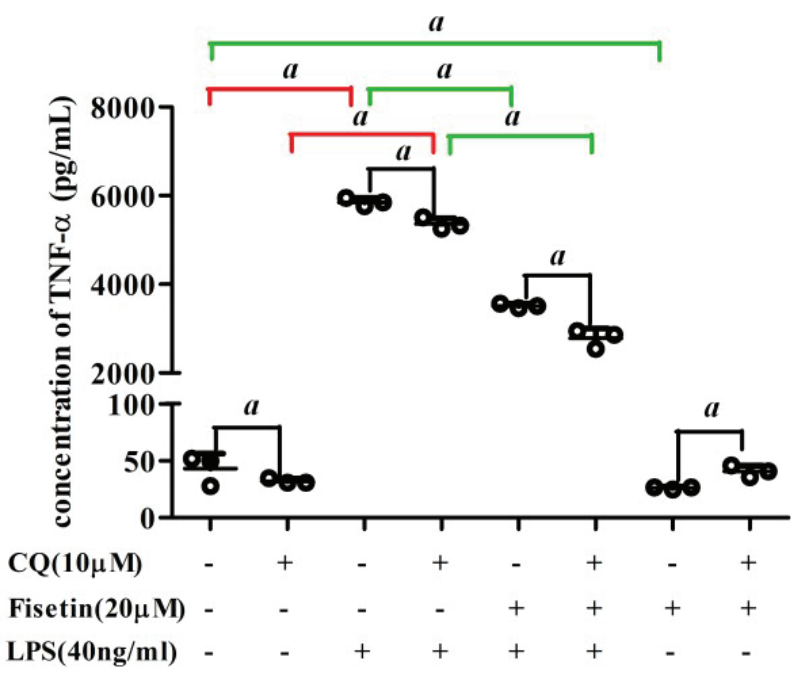

Fig. 6. Effect of CQ on the anti-inflammatory property of fisetin. Secreted proteins of (a) IL-6 and (b) TNF- $\alpha$ in cells pretreated with CQ. Data are represented as mean $\pm \mathrm{SD}(n=3) . a<0.05$.

Autophagy is an evolutionarily conserved metabolic process that is responsible for the lysosomal degradation of microorganisms, damaged organelles, and misfolded protein. Macroautophagy is the most typical form of autophagy. It begins with the formation of phagocytic assembly sites (PAS) and the assembly of the Unc-51like kinase (ULK) complex, which initiates the formation of autophagosomes. In the nucleation phase, the ULK complex interacts with the class III PI3K complex to form phagocytic vesicles. The phagocytic vesicle membrane then expands to form an autophagosome. Finally, the autophagosome fuses with a lysosome and exposes cargo to the lysosomal enzymes, leading to degradation of the content. A growing number of evidences reveal that autophagy significantly participates in the innate and adaptive immunity. First, autophagy works synergistically with pattern-recognition receptors, and acts as both a regulator and an effector of PRR signaling; second, autophagy plays a significant role in the presentation of extracellular microbial antigens on major histocompatibility complex (MHC) class II in dendritic cells and the maintenance of $\mathrm{T}$ lymphocytes homeostasis; third, autophagy can capture and eliminate intracellular microbes; finally, autophagy positively or negatively regulates the production of multiple inflammatory factors in some specific mechanisms $(19,37,38)$. In addition, it has been reported that several autophagy loci are associated with genetic predispositions for chronic inflammatory disorders and autoimmune diseases (39). The ability of fisetin to induce autophagic cell death in cancer cells has been reported in recent years. Suh et al. found that fisetin functioned as a dual inhibitor of mTORC1/2 signaling and induced autophagic cell death in PC-3 prostate cancer cells (40). Another study revealed that fisetin induced transient autophagy in response to ER stress via an AMPK-independent pathway in melanoma cells (41). Fisetin was reported to up-regulate the expression of SIRT1 and activate SIRT1 mediated deacetylation, which further decreases mTOR function and thereby induces the autophagy process (42). Fisetin can decrease $\mathrm{Pb}$-induced neuroinflammation and neurodegeneration in brains by regulating the Adenosine 5'-monophosphate-activated protein kinase (AMPK)/silence information adjustment factor 2-related enzyme 1 (SIRT1) and autophagy pathway (43). Singh et al. found that the administration of fisetin significantly up-regulated the expression of autophagy genes, such as Beclin-1 and Atg-3, in the brain of induced as well as naturally aged rats (44).

In recent years, the knowledge base and new technologies for autophagy research have been continuously studied and expanded. LC3B, a widely accepted marker for autophagic activity, is associated with autophagosome development and maturation, and is used to monitor autophagic activity (45). It is worth noting that autophagy is a dynamic process and the accumulation of autophagosomes/LC3B is not necessarily equivalent to autophagy activation. In many cases, the accumulation of autophagosomes/LC3B is due to the obstruction in trafficking of autophagosomes to lysosomes without a concomitant change in autophagosome biogenesis. LAMP1 is mainly distributed on lysosome membranes and is routinely used as a lysosome marker (46). CQ has 
been reported to inhibit the activity of the degradative enzymes by inhibiting lysosomal acidification (47). Previous studies have demonstrated that CQ acts as an autophagy late-stage inhibitor, effectively blocking lysosomal digestion and leading to the accumulation of autophagosomes $(48,49)$. This study found that LC3B and LAMP1 increased in a time-dependent manner in cells exposed to LPS, suggesting that the number of autophagosomes and lysosomes increased. After inhibiting lysosomal degradation by CQ, LC3B and LAMP1 levels were comparable in the control group and the LPS group, which indicated that the increase of autophagosomes was due to the blockade in autophagosome-lysosome fusion and degradation rather than increased production. Xia et al. proved that LPS $(20 \mathrm{ng} / \mathrm{mL})$ inhibited the expression of ATP6V0D2 in bone marrow-derived macrophages, which was a key component of V-ATPase and could promote autophagosome-lysosome membrane fusion (50). However, Tan et al. found that LPS $(100 \mu \mathrm{g} / \mathrm{mL})$ induced podocyte injury by inhibiting the early stage of autophagy with the decreased expression of LC3 and Beclin1 and the increased expression of P62 (51). Owing to the differences in cell type and LPS intervention concentration, we observed that LPS (40 $\mathrm{ng} / \mathrm{mL})$ inhibited the late stage of autophagy and led to the increased protein levels of LC3 and LAMP1 in RAW264.7 cells. Fisetin treatment decreased the number of autophagosomes and lysosomes in LPS-treated cells. The decrease of LC3B and LAMP1 in cells without CQ pretreatment was higher than that in cells with CQ pretreatment, which suggested that fisetin facilitated the autophagosome-lysosome fusion and degradation in LPS-treated cells, resulting in decreased autophagosomes and lysosomes. Notably, for CQ-pretreated cells, the increase of LC3B and LAMP1 in the fisetin group was lower than that in the control group, which indicated that fisetin might improve the inhibition of autophagosome-lysosome fusion and degradation by CQ. Jia et al. proved that fisetin-treated human pancreatic cancer PANC-1 cells show increased autophagy, which is mediated by $\mathrm{p} 8$ through the p53/PKC- $\alpha$ pathway, and combination with autophagy inhibitors can significantly strengthen the effect of fisetin (52).

mTOR, the mammalian target of rapamycin, is a pivotal negative regulatory axis of autophagy and a downstream target of the PI3K and PKA pathways. Numerous natural products have been proved to suppress cancer cells by targeting the PI3K/AKT/mTOR-mediated autophagy (53). Wang et al. found a natural source of flavonoid, sotetsuflavone, which could induce autophagy by blocking PI3K/ AKT/mTOR pathways in NSCLC cells (54). Besides, the $\mathrm{PI} 3 \mathrm{~K} / \mathrm{AmKT} / \mathrm{mTOR}$ signaling pathway is involved in the regulation of inflammation, which is usually related to the activation of TLRs/NF- $\mathrm{kB}$, cytokine receptor and tyrosine kinase receptor signals (55). Utsugi et al. found that PI3K p110 $\beta$ positively regulated IL-12 production through the JNK1-dependent pathway in human macrophages and dendritic cells (56). Furthermore, salidroside exerted a pronounced cardioprotective effect in rats subjected to LPS possibly through the inhibition of iNOS, COX-2, NF- $\kappa \mathrm{B}$ and PI3K/Akt/mTOR pathway (57). In this study, exposure of LPS could trigger phosphorylation of PI3K, AKT, and $\mathrm{mTOR}$, and fisetin could effectively inhibit this process, which suggested that PI3K/Akt/mTOR was an effective pathway for fisetin to exert anti-inflammatory effects and increase autophagic activities.

Although the defects in autophagy are involved in several inflammatory diseases, the role of autophagy in inflammatory cytokine production remains unclear. In this study, IL- 6 and TNF- $\alpha$ in the control group, the LPS group, and the fisetin and LPS co-treatment group decreased after CQ pretreatment. There are several speculations for this result. First, autophagy inhibits the production of inflammatory cytokines. Li et al. found that autophagy inhibitor 3-methyladenine (3-MA) attenuated the sepsis symptoms, as well as IL- 6 and TNF- $\alpha$ production in a lethal model of murine endotoxemia and polymicrobial sepsis, while autophagy-enhancer rapamycin increased the inflammatory damages. Further research revealed that 3-MA and rapamycin might interfere with the TLR4 signaling pathway to regulate the TNF- $\alpha$ production in LPS-activated macrophages (58). Second, the role of autophagy in the secretion of proinflammatory factors involves more unconventional secretory pathways, rather than conventional biosynthetic pathways. It was reported that autophagy had a positive contribution to the biogenesis and secretion of the proinflammatory cytokine IL- $1 \beta$ via an export pathway that depends on $\operatorname{Atg} 5$, inflammasome, at least one of the two mammalian Golgi reassembly stacking protein (GRASP) paralogues, GRASP55 and Rab8a (59). $\mathrm{Pu}$ et al. demonstrated that the loss of Atg7 led to an increased production of IL-1 $\beta$ and enhanced inflammasome activation, but IL- 6 and TNF- $\alpha$ levels were not changed (60). Third, in addition to being an autophagy inhibitor, CQ has been proved to be an effective antiinflammatory drug for rheumatic diseases (61). It could reduce the deaths induced by LPS in mice through inhibition of HMGB1 release and NF- $\mathrm{kB}-$ mediated inflammatory pathways (62). However, we found that TNF- $\alpha$ levels increased after CQ treatment in the fisetin group. The decrease of IL-6 and TNF- $\alpha$ levels was lower in the LPS group than that in the control group, and was higher in the fisetin and LPS co-treatment group than that in the LPS group. These results suggested that the inhibition or activation of autophagy was associated with the pro-/anti-inflammation effects. Specifically, fisetin exerts anti-inflammatory effects by increasing autophagy. This inference requires confirmation in further study. 


\section{Conclusions}

This research study identified 1,071 genes regulated by fisetin in LPS-treated RAW264.7 cells using a high-throughput technology. Based on the microarray analysis, we proved that fisetin inhibited the expression and secretion of inflammatory cytokines and facilitated autophagosome-lysosome fusion and degradation in LPS-treated RAW264.7 cells via the inhibition of PI3K/AKT/mTOR signaling pathway. The findings of this study suggested that fisetin was an important anti-inflammatory bioactive compound and deserved intensive scientific exploration for the prevention and treatment of inflammation-related diseases.

\section{Data availability}

Data used to support the findings of this study are available from the corresponding author (Jihua Chen) upon reasonable request.

\section{Conflict of interest and funding}

The authors declare that there is no conflict of interest regarding the publication of this article. The research study was supported by the National Natural Science Foundation of China (No. 81472972).

\section{References}

1. Wu MY, Li CJ, Hou MF, Chu PY. New insights into the role of inflammation in the pathogenesis of atherosclerosis. Int J Mol Sci 2017; 18(10): 2034. doi: 10.3390/ijms18102034

2. Fredman G, Tabas I. Boosting inflammation resolution in atherosclerosis: the next frontier for therapy. Am J Pathol 2017; 187(6): 1211-21. doi: 10.1016/j.ajpath.2017.01.018

3. Sands BE. Biomarkers of inflammation in inflammatory bowel disease. Gastroenterology 2015; 149(5): 1275-85.e2. doi: 10.1053/j. gastro.2015.07.003

4. Jonsson MK, Sundlisaeter NP, Nordal HH, Hammer HB, Aga $\mathrm{AB}$, Olsen IC, et al. Calprotectin as a marker of inflammation in patients with early rheumatoid arthritis. Ann Rheum Dis 2017; 76(12): 2031-7. doi: 10.1136/annrheumdis-2017-211695

5. Chitnis T, Weiner HL. CNS inflammation and neurodegeneration. J Clin Invest 2017; 127(10): 3577-87. doi: 10.1172/JCI90609

6. Libby P. Inflammatory mechanisms: the molecular basis of inflammation and disease. Nutr Rev 2007; 65(12 Pt 2): S140-6. doi: 10.1111/j.1753-4887.2007.tb00352.x

7. Jia G, Liu X, Che N, Xia Y, Wang G, Xiong Z, et al. Human-origin Lactobacillus salivarius AR809 protects against immunosuppression in S. aureus-induced pharyngitis via Akt-mediated NF-kappaB and autophagy signaling pathways. Food Funct 2020; 11(1): 270-84. doi: 10.1039/ c9fo02476j

8. Wynn TA, Vannella KM. Macrophages in tissue repair, regeneration, and fibrosis. Immunity 2016; 44(3): 450-62. doi: 10.1016/j. immuni.2016.02.015

9. Zhang L, Sun D, Bao Y, Shi Y, Cui Y, Guo M. Nerolidol protects against LPS-induced acute kidney injury via inhibiting TLR4/NF-kappaB signaling. Phytother Res 2017; 31(3): 45965. doi: $10.1002 /$ ptr. 5770

10. Wang J, Zhang P, He H, Se X, Sun W, Chen B, et al. Eburicoic acid from Laetiporus sulphureus (Bull.:Fr.) Murrill attenuates inflammatory responses through inhibiting LPS-induced activation of PI3K/Akt/mTOR/NF-kappaB pathways in RAW264.7 cells. Naunyn Schmiedebergs Arch Pharmacol 2017; 390(8): 845-56. doi: 10.1007/s00210-017-1382-3

11. Hao L, Zhong W, Dong H, Guo W, Sun X, Zhang W, et al. ATF4 activation promotes hepatic mitochondrial dysfunction by repressing NRF1-TFAM signalling in alcoholic steatohepatitis. Gut. Published Online First. 2020. doi: 10.1136/ gutjnl-2020-321548

12. Chen ZH, Wu YF, Wang PL, Wu YP, Li ZY, Zhao Y, et al. Autophagy is essential for ultrafine particle-induced inflammation and mucus hyperproduction in airway epithelium. Autophagy 2016; 12(2): 297-311. doi: 10.1080/15548627.2015 .1124224

13. Terasawa K, Tomabechi Y, Ikeda M, Ehara H, Kukimoto-Niino M, Wakiyama M, et al. Lysosome-associated membrane proteins-1 and -2 (LAMP-1 and LAMP-2) assemble via distinct modes. Biochem Biophys Res Commun 2016; 479(3): 489-95. doi: 10.1016/j.bbrc.2016.09.093

14. Eskelinen EL. Roles of LAMP-1 and LAMP-2 in lysosome biogenesis and autophagy. Mol Aspects Med 2006; 27(5-6): 495-502. doi: 10.1016/j.mam.2006.08.005

15. Yang Z, Klionsky DJ. An overview of the molecular mechanism of autophagy. Curr Top Microbiol Immunol 2009; 335: 1-32. doi: 10.1007/978-3-642-00302-8_1

16. Hu YX, Han XS, Jing Q. Autophagy in development and differentiation. Adv Exp Med Biol 2019; 1206: 469-87. doi: 10.1007/ 978-981-15-0602-4_22

17. Li X, He S, Ma B. Autophagy and autophagy-related proteins in cancer. Mol Cancer 2020; 19(1): 12. doi: 10.1186/ s12943-020-1138-4

18. Cadwell K. Crosstalk between autophagy and inflammatory signalling pathways: balancing defence and homeostasis. Nat Rev Immunol 2016; 16(11): 661-75. doi: 10.1038/nri.2016.100

19. Wu MY, Lu JH. Autophagy and macrophage functions: inflammatory response and phagocytosis. Cells 2019; 9(1). doi: 10.3390/ cells 9010070

20. Baldim JL, De Alcantara BGV, Domingos ODS, Soares MG, Caldas IS, Novaes RD, et al. The correlation between chemical structures and antioxidant, prooxidant, and antitrypanosomatid properties of flavonoids. Oxid Med Cell Longev 2017; 2017: 3789856. doi: 10.1155/2017/3789856

21. Hertog MG, Feskens EJ, Hollman PC, Katan MB, Kromhout D. Dietary antioxidant flavonoids and risk of coronary heart disease: the Zutphen Elderly Study. Lancet 1993; 342(8878): 1007-11. doi: 10.1016/0140-6736(93)92876-u

22. Knekt P, Jarvinen R, Reunanen A, Maatela J. Flavonoid intake and coronary mortality in Finland: a cohort study. BMJ 1996; 312(7029): 478-81. doi: 10.1136/bmj.312.7029.478

23. Knekt P, Jarvinen R, Seppanen R, Hellovaara M, Teppo L, Pukkala E, et al. Dietary flavonoids and the risk of lung cancer and other malignant neoplasms. Am J Epidemiol 1997; 146(3): 223-30. doi: 10.1093/oxfordjournals.aje.a009257

24. Hertog MG, Feskens EJ, Hollman PC, Katan MB, Kromhout D. Dietary flavonoids and cancer risk in the Zutphen Elderly Study. Nutr Cancer 1994; 22(2): 175-84. doi: 10.1080/01635589409514342

25. Keli SO, Hertog MG, Feskens EJ, Kromhout D. Dietary flavonoids, antioxidant vitamins, and incidence of stroke: the Zutphen study. Arch Intern Med 1996; 156(6): 637-42.

26. Pal HC, Pearlman RL, Afaq F. Fisetin and its role in chronic diseases. Adv Exp Med Biol 2016; 928: 213-44. doi: 10.1007/978-3-319-41334-1_10 
27. Gelderblom M, Leypoldt F, Lewerenz J, Birkenmayer G, Orozco D, Ludewig P, et al. The flavonoid fisetin attenuates postischemic immune cell infiltration, activation and infarct size after transient cerebral middle artery occlusion in mice. $\mathrm{J}$ Cereb Blood Flow Metab 2012; 32(5): 835-43. doi: 10.1038/ jcbfm.2011.189

28. Kim SC, Kang SH, Jeong SJ, Kim SH, Ko HS, Kim SH. Inhibition of c-Jun $\mathrm{N}$-terminal kinase and nuclear factor kappa B pathways mediates fisetin-exerted anti-inflammatory activity in lipopolysccharide-treated RAW264.7 cells. Immunopharmacol Immunotoxicol 2012; 34(4): 645-50. doi: 10.3109/08923973.201 1.648270

29. Wang Y, Wang L, Wise JTF, Shi X, Chen Z. Verteporfin inhibits lipopolysaccharide-induced inflammation by multiple functions in RAW 264.7 cells. Toxicol Appl Pharmacol 2020; 387: 114852. doi: 10.1016/j.taap.2019.114852

30. Kim JH, Kim MY, Kim JH, Cho JY. Fisetin suppresses macrophage-mediated inflammatory responses by blockade of Src and Syk. Biomol Ther (Seoul). 2015; 23(5): 414-20. doi: 10.4062/ biomolther.2015.036

31. Lyu SY, Park WB. Production of cytokine and NO by RAW 264.7 macrophages and PBMC in vitro incubation with flavonoids. Arch Pharm Res 2005; 28(5): 573-81. doi: 10.1007/bf02977761

32. Sahu BD, Kumar JM, Sistla R. Fisetin, a dietary flavonoid, ameliorates experimental colitis in mice: relevance of NF-kappaB signaling. J Nutr Biochem 2016; 28: 171-82. doi: 10.1016/j. jnutbio.2015.10.004

33. Feng G, Jiang ZY, Sun B, Fu J, Li TZ. Fisetin alleviates lipopolysaccharide-induced acute lung injury via TLR4-mediated NF-kappaB signaling pathway in rats. Inflammation 2016; 39(1): 148-57. doi: 10.1007/s10753-015-0233-y

34. Ahmad A, Ali T, Rehman SU, Kim MO. Phytomedicine-based potent antioxidant, fisetin protects CNS-insult LPS-induced oxidative stress-mediated neurodegeneration and memory impairment. J Clin Med 2019; 8(6): 850. doi: 10.3390/ jcm8060850

35. Li P, Chen D, Huang Y. Fisetin administration improves LPSinduced acute otitis media in mouse in vivo. Int J Mol Med 2018; 42(1): 237-47. doi: 10.3892/ijmm.2018.3585

36. Iyer SS, Cheng G. Role of interleukin 10 transcriptional regulation in inflammation and autoimmune disease. Crit Rev Immunol 2012; 32(1): 23-63. doi: 10.1615/critrevimmunol. v32.11.30

37. Castillo EF, Dekonenko A, Arko-Mensah J, Mandell MA, Dupont N, Jiang S, et al. Autophagy protects against active tuberculosis by suppressing bacterial burden and inflammation. Proc Natl Acad Sci USA 2012; 109(46): E3168-76. doi: 10.1073/ pnas. 1210500109

38. Levine B, Mizushima N, Virgin HW. Autophagy in immunity and inflammation. Nature 2011; 469(7330): 323-35. doi: 10.1038/ nature09782

39. Deretic V, Levine B. Autophagy balances inflammation in innate immunity. Autophagy 2018; 14(2): 243-51. doi: 10.1080/155486 27.2017.1402992

40. Suh Y, Afaq F, Khan N, johnson JJ, Khusro FH, Mukhtar H. Fisetin induces autophagic cell death through suppression of mTOR signaling pathway in prostate cancer cells. Carcinogenesis 2010; 31(8): 1424-33. doi: 10.1093/carcin/bgq115

41. Syed DN, Lall RK, Chamcheu JC, Haidar O, Mukhtar H. Involvement of ER stress and activation of apoptotic pathways in fisetin induced cytotoxicity in human melanoma. Arch Biochem Biophys 2014; 563: 108-17. doi: 10.1016/j. abb.2014.06.034
42. Syed DN, Adhami VM, Khan MI, Mukhtar H. Inhibition of Akt/mTOR signaling by the dietary flavonoid fisetin. Anticancer Agents Med Chem 2013; 13(7): 995-1001. doi: 10.2174/187152061 13139990129

43. Yang W, Tian ZK, Yang HX, Feng ZJ, Sun JM, Jiang H, et al. Fisetin improves lead-induced neuroinflammation, apoptosis and synaptic dysfunction in mice associated with the AMPK/ SIRT1 and autophagy pathway. Food Chem Toxicol 2019; 134: 110824. doi: 10.1016/j.fct.2019.110824

44. Singh S, Singh AK, Garg G, Rizvi SI. Fisetin as a caloric restriction mimetic protects rat brain against aging induced oxidative stress, apoptosis and neurodegeneration. Life Sci 2018; 193: 171-9. doi: 10.1016/j.lfs.2017.11.004

45. Schaaf MB, Keulers TG, Vooijs MA, Rouschop KM. LC3/ GABARAP family proteins: autophagy-(un)related functions. FASEB J 2016; 30(12): 3961-78. doi: 10.1096/fj.201600698R

46. Cheng XT, Xie YX, Zhou B, Huang N, Farfel-Becker T, Sheng $\mathrm{ZH}$. Revisiting LAMP1 as a marker for degradative autophagy-lysosomal organelles in the nervous system. Autophagy 2018; 14(8): 1472-4. doi: 10.1080/15548627.2018.1482147

47. Homewood CA, Warhurst DC, Peters W, Baggaley VC. Lysosomes, $\mathrm{pH}$ and the anti-malarial action of chloroquine. Nature 1972; 235(5332): 50-2. doi: 10.1038/235050a0

48. Amaravadi RK, Yu D, Lum JJ, Bui T, Christophorou MA, Evan GI, et al. Autophagy inhibition enhances therapy-induced apoptosis in a Myc-induced model of lymphoma. J Clin Invest 2007; 117(2): 326-36. doi: 10.1172/JCI28833

49. Fan QW, Cheng C, Hackett C, Feldman M, Houseman BT, Nicolaides T, et al. Akt and autophagy cooperate to promote survival of drug-resistant glioma. Sci Signal 2010; 3(147): ra81. doi: 10.1126/scisignal.2001017

50. Xia Y, Liu N, Xie X, Bi G, Ba H, Li L, et al. The macrophage-specific V-ATPase subunit ATP6V0D2 restricts inflammasome activation and bacterial infection by facilitating autophagosome-lysosome fusion. Autophagy 2019; 15(6): 960-75. doi: 10. 1080/15548627.2019.1569916

51. Tan X, Chen Y, Liang X, Yu C, Lai Y, zhang L, et al. Lipopolysaccharide-induced podocyte injury is mediated by suppression of autophagy. Mol Med Rep 2016; 14(1): 811-8. doi: 10.3892/mmr.2016.5301

52. Jia S, Xu X, Zhou S, Chen Y, Ding G, Cao L. Fisetin induces autophagy in pancreatic cancer cells via endoplasmic reticulum stress- and mitochondrial stress-dependent pathways. Cell Death Dis 2019; 10(2): 142. doi: 10.1038/s41419-019-1366-y

53. Xu Z, Han X, Ou D, Liu T, Li Z, Jiang G, et al. Targeting $\mathrm{PI} 3 \mathrm{~K} / \mathrm{AKT} / \mathrm{mTOR}$-mediated autophagy for tumor therapy. Appl Microbiol Biotechnol 2020; 104(2): 575-87. doi: $10.1007 / \mathrm{s} 00253-019-10257-8$

54. Wang S, Xu X, Hu Y, Lei T, Liu T. Sotetsuflavone induces autophagy in non-small cell lung cancer through blocking PI3K/Akt/ mTOR signaling pathway in vivo and in vitro. Front Pharmacol 2019; 10: 1460. doi: 10.3389/fphar.2019.01460

55. Saxton RA, Sabatini DM. mTOR signaling in growth, metabolism, and disease. Cell 2017; 169(2): 361-71. doi: 10.1016/j. cell.2017.03.035

56. Utsugi M, Dobashi K, Ono A, Ishizuka T, Matsuzaki S, Hisada T, et al. PI3K p110beta positively regulates lipopolysaccharide-induced IL-12 production in human macrophages and dendritic cells and JNK1 plays a novel role. J Immunol 2009; 182(9): 5225-31. doi: 10.4049/jimmunol.0801352

57. Chen L, Liu P, Feng X, Ma C. Salidroside suppressing LPSinduced myocardial injury by inhibiting ROS-mediated PI3K/ Akt/mTOR pathway in vitro and in vivo. J Cell Mol Med 2017; 21(12): 3178-89. doi: $10.1111 /$ jcmm.12871 
58. Li Q, Li L, Fei X, Zhang Y, Qi C, Hua S, et al. Inhibition of autophagy with 3-methyladenine is protective in a lethal model of murine endotoxemia and polymicrobial sepsis. Innate Immun 2018; 24(4): 231-9. doi: 10.1177/1753425918771170

59. Dupont N, Jiang S, Pilli M, Ornatowski W, Bhattacharya D, Deretic V. Autophagy-based unconventional secretory pathway for extracellular delivery of IL-1beta. EMBO J 2011; 30(23): 4701-11. doi: 10.1038/emboj.2011.398

60. Pu Q, Gan C, Li R, Li Y, Tan S, Li X, et al. Atg7 deficiency intensifies inflammasome activation and pyroptosis in pseudomonas sepsis. J Immunol 2017; 198(8): 3205-13. doi: 10.4049/jimmunol.1601196

61. Ben-Zvi I, Kivity S, Langevitz P, Shoenfeld Y. Hydroxychloroquine: from malaria to autoimmunity. Clin Rev Allergy Immunol 2012; 42(2): 145-53. doi: 10.1007/s12016-010-8243-x
62. Yang M, Cao L, Xie M, Yu Y, Kang R, Yang L, et al. Chloroquine inhibits HMGB1 inflammatory signaling and protects mice from lethal sepsis. Biochem Pharmacol 2013; 86(3): 410-8. doi: 10.1016/j.bcp.2013.05.013

\section{*Jihua Chen}

Department of Nutrition Science and Food Hygiene, Xiangya School of Public Health,

Central South University,

I 10 Xiangya Road, Changsha, Hunan 410078

China

Email: chenjh@csu.edu.cn 